\title{
Spontaneous breaking of gauge groups to discrete symmetries
}

\author{
Bradley L. Rachlin and Thomas W. Kephart \\ Department of Physics and Astronomy, Vanderbilt University, \\ Nashville, TN 37235, U.S.A. \\ E-mail: bradleyrachlin@gmail.com, tom.kephart@gmail.com
}

ABSTRaCT: Many models of beyond Standard Model physics connect flavor symmetry with a discrete group. Having this symmetry arise spontaneously from a gauge theory maintains compatibility with quantum gravity and can be used to systematically prevent anomalies. We minimize a number of Higgs potentials that break gauge groups to discrete symmetries of interest, and examine their scalar mass spectra.

Keywords: Beyond Standard Model, Discrete Symmetries, Gauge Symmetry, Spontaneous Symmetry Breaking

ArXiv EPRINT: 1702.08073 


\section{Contents}

1 Introduction 1

$\begin{array}{lll}2 & \text { Lie group invariant potentials } & \mathbf{2}\end{array}$

2.1 Gauge group irreps containing discrete gauge singlets 2

$2.2 \mathrm{SO}(3)$ potentials 3

2.2.1 $A_{4} \quad 3$

$\begin{array}{lll}2.2 .2 & S_{4} & 4\end{array}$

2.2.3 $A_{5} \quad 4$

$2.3 \mathrm{SU}(2)$ potentials $\quad 5$

2.3.1 $Q_{6} \quad 5$

$\begin{array}{lll}2.3 .2 & T^{\prime} & 5\end{array}$

$2.3 .3 \quad O^{\prime} \quad 5$

$2.3 .4 \quad I^{\prime} \quad 6$

$\begin{array}{lll}2.4 & \mathrm{SU}(3) \text { potentials } & 6\end{array}$

$\begin{array}{lll}2.4 .1 & A_{4} \text { and } T_{7} & 7\end{array}$

$\begin{array}{lll}2.4 .2 & \Delta(27) & 7\end{array}$

$\begin{array}{lll}2.4 .3 & \operatorname{PSL}(2,7) & 7\end{array}$

3 Vaccuum alignments for spontaneous symmetry breaking $\quad 8$

$\begin{array}{lll}3.1 & \text { Vacuua for } \mathrm{SO}(3) \text { potentials } & 8\end{array}$

$\begin{array}{llr}3.1 .1 & A_{4} & 9\end{array}$

$\begin{array}{lll}3.1 .2 & S_{4} & 10\end{array}$

$\begin{array}{lll}3.1 .3 & A_{5} & 10\end{array}$

$\begin{array}{ll}3.2 \text { Vacuua for } \mathrm{SU}(2) \text { potentials } & 11\end{array}$

$\begin{array}{lll}3.2 .1 & T^{\prime} & 11\end{array}$

$\begin{array}{lll}3.2 .2 & Q_{6} & 12\end{array}$

$\begin{array}{lll}3.2 .3 & O^{\prime} & 12\end{array}$

$\begin{array}{lll}3.2 .4 \quad I^{\prime} & 13\end{array}$

$\begin{array}{lll}3.3 & \text { Vacuua for } \mathrm{SU}(3) \text { potentials } & 13\end{array}$

$\begin{array}{lll}3.3 .1 & A_{4} & 14\end{array}$

$\begin{array}{lll}3.3 .2 & T_{7} & 14\end{array}$

$\begin{array}{lll}3.3 .3 & \Delta(27) & 15\end{array}$

$\begin{array}{lll}3.3 .4 & \operatorname{PSL}(2,7) & 15\end{array}$

4 Vacuum expectation values and mass spectra $\quad 17$

$\begin{array}{lll}4.1 & \mathrm{SO}(3) \text { cases } & 17\end{array}$

$\begin{array}{lll}\text { 4.1.1 } & A_{4} \text { scalar spectrum } & 17\end{array}$

$\begin{array}{lll}4.1 .2 & S_{4} \text { scalar spectrum } & 18\end{array}$

$\begin{array}{lll}\text { 4.1.3 } & A_{5} \text { scalar spectrum } & 18\end{array}$

$\begin{array}{lll}4.2 & \mathrm{SU}(2) \text { cases } & 19\end{array}$ 
4.2.1 $Q_{6}$ scalar spectrum $\quad 19$

4.2.2 $T^{\prime}$ scalar spectrum 20

4.2.3 $O^{\prime}$ scalar spectrum $\quad 20$

$\begin{array}{lll}4.2 .4 & I^{\prime} \text { scalar spectrum } & 21\end{array}$

$\begin{array}{lll}4.3 & \mathrm{SU}(3) \text { cases } & 22\end{array}$

4.3.1 $A_{4}$ scalar spectrum 22

4.3.2 $T_{7}$ scalar spectrum 23

$\begin{array}{lll}4.3 .3 & \Delta(27) \text { scalar spectrum } & 24\end{array}$

4.3.4 PSL(2,7) scalar spectrum 24

$\begin{array}{lll}4.4 & \text { Symmetry breaking summary } & 26\end{array}$

$\begin{array}{llr}5 & \text { Discussion and conclusion } & 27\end{array}$

$\begin{array}{ll}\text { A Branching rules } & 29\end{array}$

\section{Introduction}

The standard model (SM) does not explain quark and lepton masses, nor does it explain how quarks and leptons mix. The most studied and perhaps the most aesthetic approach to parameterizing the masses and mixing angles is to extend the SM by a discrete group $\Gamma$ into whose irreducible representations (irreps) the standard model particles are assigned. Many choices of this discrete flavor symmetry have been tried. As expected, larger groups can typically provide a fuller description of flavor physics, but there are examples of relatively small nonabelian discrete groups like $A_{4}$ and $T^{\prime}$ that are somewhat more economical. Here we take an agnostic approach as to the choice of discrete group and study a representative set of examples that have been used in model building.

Notable early extensions of the standard model with discrete symmetries include the work of Pakvasa and Sugawara [1] and Wyler [2] who used $\Gamma=S_{3}$ and $\Gamma=A_{4}$ respectively to describe the quark sector, as well as Ma and collaborators [3, 4] who used $\Gamma=A_{4}$ to describe the lepton sector. Many other choices for $\Gamma$ have subsequently been used in model building, several of which will be discussed below. For an early brief review of possible discrete groups that can be used for SM extensions see [5]. Recent extensive reviews with more complete and up to date bibliographies are also available. See for instance [6-9].

Extending the SM by a discrete group is not without its perils. Global discrete symmetries are violated by gravity [10]. (As an example, consider the case when a star collapses to a black hole. The no hair theorem tells us initial baryon number is lost and hence gravity causes a global discrete symmetry to be violated. Similarly, global continuous symmetries are violated by gravity. See e.g., [11-13], where it is argued that gravity also spoils the Peccei-Quinn solution to the strong CP problem.) In addition, the discrete group can be anomalous [14], it can lead to unwanted cosmic defects [15], etc. To avoid as many of these problems as possible the most expedient approach is to gauge the discrete symmetry, i.e., 
extend the SM by a continuous gauge group $G$ in such a way that no chiral anomalies are produced. Then one breaks this gauge group to the desired discrete group, $G \rightarrow \Gamma$, where now $\Gamma$ is effectively anomaly free and avoids problems with gravity.

Various examples of Lie groups breaking to discrete groups have been discussed in the literature, but only in a few cases have the details of the minimization of the scalar potential and the extraction of the scalar spectrum been investigated. Here we plan to include these important details for many of the discrete groups of interest via the following procedure:

(i) First we provide irreps of $G$ that contain trivial $\Gamma$ singlets. These results are summarized in the appendix.

(ii) Next we set up scalar potentials $V$ with scalars in one of these irreps.

(iii) Then we find a vacuum expectation value (VEV) via the Reynolds operator $[16,17]$ (related to the perhaps more familiar Molien series [18]) that can break $G$ to $\Gamma$.

(iv) Next we minimize $V$ to show that the VEV indeed does properly break the symmetry.

(v) Finally, we provide the spectrum of scalar masses at the $\Gamma$ level after the breaking.

Our calculations are carried out with Mathematica and checked by hand where practical.

Many of the methods we employ were developed in work by Luhn [19] and by Merle and Zwicky [20], where some of the results summarized here can be found. We believe our results will be of interest to many model builders, since it will allow them to include the minimal set of scalars necessary to break a gauge symmetry to a discrete symmetry of interest. A few examples that go beyond the minimal set of scalars are also included, where the symmetry breaking is carried out from a nonminimal $G$ irrep or from a non-minimal $G$.

\section{$2 \quad$ Lie group invariant potentials}

Our task in this section is to construct Higgs potentials invariant under Lie groups $G$ for specific irreps. But first we must see which irreps are suitable for spontaneous symmetry breaking (SSB), i.e., irreps whose decompositions include a trivial singlet of the desired subgroup $\Gamma \subset G$ to which we hope to break. Using the Mathematica package decomposeLGreps [21] along with GAP to generate the groups [22], one can easily produce tables of branching rules from Lie group irreps to subgroup irreps and find such singlets. We have done this for a number of cases and have included them in a short appendix for convenience and to make the paper self contained.

\subsection{Gauge group irreps containing discrete gauge singlets}

The discrete groups $\Gamma$ we will discuss and the gauge groups where they can be minimally embedded are $A_{4}, S_{4}, A_{5} \subset \mathrm{SO}(3) ; Q_{6}, T^{\prime}, O^{\prime}, I^{\prime} \subset \mathrm{SU}(2)$; and $T_{7}, \Delta(27), \operatorname{PSL}(2,7) \subset \mathrm{SU}(3)$.

Some of these discrete groups can also be embedded non-minimally. For example, we include the case $A_{4} \subset \mathrm{SU}(3)$. Minimal and non-minimal embedding of other discrete groups can be handled in a way similar to what is discussed here, and we hope that the examples we discuss are sufficiently informative to aid in other cases. 
To spontaneously break $G$ to $\Gamma$ with some irrep $R$ of $G$, it is necessary that $R$ contains a trivial $\Gamma$ singlet. It is straightforward to look at the decomposition of $R$ from $G$ to $\Gamma$ to make this determination. The decomposition can be carried out by standard techniques starting from character tables. Since it is the character tables that are usually provided in the literature, for convenience we here provide an appendix with the tables of decompositions of the first few irreps of $\mathrm{SO}(3), \mathrm{SU}(2)$, and $\mathrm{SU}(3)$ to discrete groups of interest. For example, as one can see in table 17 of the appendix, the 7 and 9 dimensional irreps of $\mathrm{SO}(3)$ have trivial $A_{4}$ singlets, therefore these irreps are both candidates for the scalar potential that allows the spontaneous symmetry breaking $\mathrm{SO}(3) \rightarrow A_{4}$.

\section{$2.2 \mathrm{SO}(3)$ potentials}

Before going into constructing specific potentials we first comment on possible cubic terms; a general renormalizable potential has quadratic, cubic, and quartic terms, but the cubic terms tend to significantly complicate the analysis. We exclude these terms for simplicity by imposing a $Z_{2}$ symmetry (or, in many cases they vanish on their own upon summation), so the following potentials are actually $\mathrm{SO}(3) \times Z_{2}$ invariant. (The $Z_{2}$ symmetry can be avoided by including allowed cubic terms or by gauging it too.) The effect of including the cubic terms is studied for a case where the analysis is tractable in section 4 . We now proceed to our first example, the breaking pattern $\mathrm{SO}(3) \rightarrow A_{4}$.

\subsection{1 $\quad A_{4}$}

We begin by constructing an $\mathrm{SO}(3)$ invariant potential. As stated above, which irrep we use depends on the discrete subgroup of interest. For example, if we want to break to the tetrahedral group $A_{4}$, which has been used to describe the tri-bimaximal neutrino mixing pattern [7] [24] and co-bimaximal mixing [25], we look at table 17 and see that the lowest dimensional irrep we can use is the $\mathbf{7}$. (For references to other recent work with $A_{4}$ models see [26-28].) In terms of the fundamental $\mathbf{3}$ of $\mathrm{SO}(3)$, we obtain a $\mathbf{7}$ as a direct product of three $3 \mathrm{~s}$.

$$
\mathbf{3} \times \mathbf{3} \times \mathbf{3}=\mathbf{1}+3 \cdot \mathbf{3}+2 \cdot \mathbf{5}+\mathbf{7}
$$

This product gives a generic rank 3 tensor with 27 independent components. To isolate the $\mathbf{7}$, we take only the totally symmetric part, which reduces the number of components from 27 to 10, giving the symmetric tensor $S_{i j k}$. Then, using the fact that the Kroenecker delta $\delta_{i j}$, is an invariant of the fundamental irrep of $S O$ groups (for a discussion of Lie group invariant tensors see [29]) we subtract off the three traces, $\sum_{j}^{3} \delta_{j k} S_{i j k}, \mathrm{i}=1,2,3$, to obtain the traceless symmetric tensor $T_{i j k}$, which is our 7 dimensional $\mathrm{SO}(3)$ irrep. As mentioned above, the most general renormalizable potential is constructed from the independent quadratic, cubic, and quartic contractions of this tensor. In this case there are two quartic terms, but notice that all the cubic terms, which necessarily include the anti-symmetric Levi-Civita Tensor, $\epsilon_{i j k}$, vanish upon summation. Hence the potential for the 7 is

$$
V_{7}=-m^{2} T_{i j k} T_{i j k}+\lambda\left(T_{i j k} T_{i j k}\right)^{2}+\kappa T_{i j m} T_{i j n} T_{k l n} T_{k l m}
$$

It is instructive to go through the details of the construction of this potential. Others follow via similar analyses. To construct $V_{7}$ consider first the product

$$
7 \times 7=1_{\mathrm{S}}+3_{\mathrm{A}}+5_{\mathrm{S}}+7_{\mathrm{A}}+\mathbf{9}_{\mathrm{S}}+11_{\mathrm{A}}+13_{\mathrm{S}}
$$


Since the potential contains only one $\mathbf{7}$, we only need to consider the symmetric part of the tensor product that we represent schematically as

$$
T \otimes T \sim T_{i j k} T_{i j k}+T_{i j m} T_{i j n}+T_{i k m} T_{i l n}+T_{k m p} T_{l n q} .
$$

The first term is a singlet, i.e., it has no free indices, and therefore contributes to the mass term in (2.2). There is no term in (2.4) with three free indices, so we can not construct a cubic term for $V_{7}$. To find the quartic terms we need to study the symmetric part of $T \otimes T \otimes T \otimes T$. Clearly $\left(T_{i j k} T_{i j k}\right)^{2}$ is allowed and it contributes to the $\lambda$ term in (2.2). Likewise $T_{i j m} T_{i j n} T_{i^{\prime} j^{\prime} m} T_{i^{\prime} j^{\prime} n}$ contributes to the $\kappa$ term. Squaring the last two terms in (2.4) to form singlets does not give new terms, e.g., $T_{i k m} T_{i l n} T_{i^{\prime} k m} T_{i^{\prime} l n}$ again tributes to the $\kappa$ term. Hence the most general potential for a $\mathbf{7}$ of $\mathrm{SO}(3)$ is as given in eq. (2.2).

In subsequent sections we find a vector (in a particular basis) pointing in the $A_{4}$ direction, then minimize the potential, find the mass eigenstates, and show that they can all be positive, implying a stable minimum. Minimization implies certain constraints on the coupling constants must be satisfied as will be discussed. We proceed in analogous fashion for other $G \rightarrow \Gamma$ cases, but first we will collect all the potentials we need for the purpose.

\section{$2.2 .2 \quad S_{4}$}

To break to the octahedral group, $S_{4}$, we see from table 18 that the lowest irrep we can use is the 9. From examining Kroenecker products, we see that we must begin with the direct product of four 3s. Similar to the results in the previous subsection, we take the symmetric part of this rank 4 tensor, $S_{i j k l}$, which reduces the number of components to 15 . We then subtract off the six trace elements, $\sum \delta_{k l} S_{i j k l}$, to obtain the desired 9-component tensor. The associated potential is

$$
\begin{aligned}
V_{9}= & -m^{2} T_{i j k l} T_{i j k l}+\lambda\left(T_{i j k l} T_{i j k l}\right)^{2}+\kappa T_{i j k l} T_{i j k p} T_{m n o p} T_{m n o l} \\
& +\rho T_{i j k l} T_{i j o p} T_{m n o p} T_{m n k l}+\tau T_{i j k l} T_{i j m n} T_{k m o p} T_{\text {lnop }} .
\end{aligned}
$$

The construction of $V_{9}$ is slightly more complicated do to the extra tensor index on the $\mathbf{9}$, but proceeds in analogy with the construction of $V_{7}$. In fact all other potentials constructed from totally symmetric tensor irreps follow the same pattern. This covers all the cases we will consider with the exception of the $\mathbf{1 5}$ of $\mathrm{SU}(3)$ which is similar, but where the mixed symmetry of the $\mathbf{1 5}$ must be taken into account. Details of that construction can be found in [19]. For examples where the octahedral group has been used to build models see [30, 31].

\subsection{3 $\quad A_{5}$}

Another subgroup of interest, which has been used in a number of recent models [32-35], is $A_{5}$. From table 19 we see that the $\mathbf{1 3}$ is the lowest dimensional irrep that contains a trivial $A_{5}$ singlet. Again starting from the fundamental $\mathrm{SO}(3)$ triplet one can show that the Kroenecker product of six $3 \mathrm{~s}$ is needed to get an irrep of this dimension. The symmetric part of this rank 6 tensor, $S_{i j k l m n}$ has 28 independent components, which is then reduced to 13 by subtracting off the 15 trace elements, $\sum \delta_{m n} S_{i j k l m n}$. The potential

$$
\begin{aligned}
V_{13}= & -m^{2} T_{i j k l m n} T_{i j k l m n}+\lambda\left(T_{i j k l m n} T_{i j k l m n}\right)^{2}+\kappa T_{i j k l m n} T_{i j k l m t} T_{\text {opqrsn }} T_{\text {opqrst }} \\
& +\rho T_{i j k l m n} T_{i j k l s t} T_{\text {opqrmn }} T_{\text {opqrst }}+\tau T_{i j k l m n} T_{i j k r s t} T_{\text {opqlmn }} T_{\text {opqrst }},
\end{aligned}
$$

is constructed in a fashion similar to the $A_{4}$ case. 


\section{$2.3 \mathrm{SU}(2)$ potentials}

We now proceed in a similar vein to construct $\mathrm{SU}(2)$ invariant potentials. In fact, for the odd dimensional (real) representations, invariants must be constructed from triplets which furnish an unfaithful representation of $\mathrm{SU}(2) .{ }^{1}$ As such the true symmetry of the theory is not given by the potential alone and must be determined from the specifics of the model, i.e., from the full Lagrangian. In the following cases, the omission of the cubic terms means the potentials have a $\mathrm{SU}(2) \times \mathrm{U}(1)$ symmetry, where the $\mathrm{U}(1)$ is a phase. This phase can also be gauged and then broken if necessary to avoid problems with global symmetries, or in some cases cubic terms can be added that do not respect the $\mathrm{U}(1)$.

\subsection{1 $Q_{6}$}

If we want to break to $Q_{6}$ we see from table 20 that the lowest dimensional irrep we can use is the $\mathbf{5}$. However, as explained in [36, 37], this irrep will actually break to the continuous subgroup Pin(2). So we must look at the next lowest irrep with a trivial SU(2) singlet, the 7. We cannot break with a real $\mathbf{7}$ as in eq. (2.2) because there are no triplet representations of $Q_{6}$ that can be used to find a VEV in the unfaithful $\mathrm{SO}(3)$ representation. Thus we must use the complex $\mathbf{7}$, which has the same potential as needed for the $T^{\prime}$ case which is given in eq. (2.7) below.

\subsection{2 $T^{\prime}$}

To break from $\mathrm{SU}(2)$ to $T^{\prime}$, the binary tetrahedral group, we see from table 21 that the smallest $\mathrm{SU}(2)$ irrep we can use is the $\mathbf{7}$. Since we must construct it from triplets the potential is the same as in equation (2.2). The VEVs will also be the same.

Another possibility is to do the breaking to $T^{\prime}$ with a complex $\mathbf{7}$, which can be thought of as a pair of real $7 \mathrm{~s}$. We can now build our representation out of the fundamental doublets of $\mathrm{SU}(2)$, where we get the $\mathbf{7}$ by taking the direct product of six $2 \mathrm{~s}$ and isolating the tensor symmetric on all indices. The potential is

$$
\begin{aligned}
V_{7_{c}}= & -m^{2} T_{i j k l m n} T^{i j k l m n}+\lambda\left(T_{i j k l m n} T^{i j k l m n}\right)^{2}+\kappa T_{i j k l m n} T^{i j k l m t} T_{\text {opqrst }} T^{\text {opqrsn }} \\
& +\rho T_{i j k l m n} T^{i j k l s t} T_{\text {opqrst }} T^{\text {opqrmn }}+\tau T_{i j k l m n} T^{i j k r s t} T_{\text {opqrst }} T^{\text {opqlmn }} .
\end{aligned}
$$

where the indices now run from 1 to 2 . All cubic terms have vanished upon summation. $T^{\prime}$ models are economical and have been used to explain both quark and lepton sector parameters [5, 38-44]. A more complete set of recent $T^{\prime}$ model references can be found in [44].

\subsection{3 $O^{\prime}$}

To break from $\mathrm{SU}(2)$ to $O^{\prime}$, the binary octahedral group, we see from table 22 that the smallest $\mathrm{SU}(2)$ irrep we can use is the $\mathbf{9}$. As in the $S_{4}$ example, we can construct our

\footnotetext{
${ }^{1}$ Group Theory Comments: the tetrahedral group $A_{4} \subset \mathrm{SO}(3)$ has double-valued representations that correspond to single-valued representations of the binary (double) tetrahedral group $T^{\prime} \subset \mathrm{SU}(2)$. As $\mathrm{SO}(3)$ is not a subgroup of $\mathrm{SU}(2)$, likewise $A_{4}$ is not a subgroup of $T^{\prime}$ [23]. Hence, besides the irreps of $T^{\prime}$ that are coincident with those of $A_{4}, T^{\prime}$ has three additional spinor doublet-like irreps. The relationships between $S_{4}$ and $O^{\prime}$ and between $A_{5}$ and $I^{\prime}$ are similar.
} 
potential from triplets so the potential is the same as in equation (2.5) and the VEVs will again be the same.

We can also consider the case of a complex $\mathbf{9}$ and build the representation out of SU(2) doublets. We obtain the $\mathbf{9}$ through the symmetric product of eight $\mathbf{2 s}$. The potential is

$$
\begin{aligned}
V_{9_{c}}= & -m^{2} T_{i j k l m n o p} T^{i j k l m n o p}+\lambda\left(T_{i j k l m n o p} T^{i j k l m n o p}\right)^{2} \\
& +\kappa T_{i j k l m n o p} T^{i j k l m n o x} T_{\text {qrstuvwx }} T^{\text {qrstuvwp }}+\rho T_{i j k l m n o p} T^{i j k l m n w x} T_{\text {qrstuvwx }} T^{\text {qrstuvop }} \\
& +\tau T_{i j k l m n o p} T^{i j k l m v w x} T_{\text {qrstuvwx }} T^{\text {qrstunop }}+\sigma T_{i j k l m n o p} T^{\text {ijkluvwx }} T_{\text {qrstuvwx }} T^{\text {qrstmnop }} .
\end{aligned}
$$

\subsection{4 $\quad I^{\prime}$}

The final $\mathrm{SU}(2)$ breaking case we consider is $I^{\prime}$, the binary icosahedral group, which has been used in both three and four family extensions of the SM $[45,46]$. Here the lowest $\mathrm{SU}(2)$ irrep we can use is the real 13, which yields the same potential as we used for $A_{5}$ (eq. (2.6)).

Alternatively for the case of a complex $\mathbf{1 3}$ we see that it is given by the symmetric product of twelve $2 \mathrm{~s}$. The potential has seven quartic invariants, and the first few terms are

$$
\begin{aligned}
V_{13_{c}=} & -m^{2} T_{\text {abcdefghijkl }} T^{\text {abcdefghijkl }}+\lambda\left(T_{\text {abcdefghijkl }} T^{\text {abcdefghijkl }}\right)^{2} \\
& +\kappa T_{\text {abcdefghijkl }} T^{\text {abcdefghijkx }} T_{\text {mnopqrstuvwx }} T^{\text {mnopqrstuvwl }}+\ldots
\end{aligned}
$$

Potentials for higher tensors can be cumbersome to write, so let us introduce a new notation to deal with them. For instance for the potential for the 13, let us define

$$
T_{12 a} \cdot T^{12 a}=T_{a b c d e f g h i j k l} T^{a b c d e f g h i j k l},
$$

and

$$
\left(T_{11 a} \cdot T^{11 a}\right)_{c}^{b}\left(T_{11 a} \cdot T^{11 a}\right)_{b}^{c}=T_{a b c d e f g h i j k l} T^{a b c d e f g h i j k x} T_{m n o p q r s t u v w x} T^{\text {mnopqrstuvwl }},
$$

etc. Specifically we write $n a$ for the collection of indices $a_{1} a_{2} a_{3} \ldots a_{n}$, etc. Then the full potential is for the complex $\mathbf{1 3}$ takes the form

$$
\begin{aligned}
V_{13_{c}}= & -m^{2} T_{12 a} \cdot T^{12 a}+\lambda\left(T_{12 a} \cdot T^{12 a}\right)^{2}+\kappa\left(T_{11 a} \cdot T^{11 a}\right)_{c}^{b}\left(T_{11 a} \cdot T^{11 a}\right)_{b}^{c} \\
& +\rho\left(T_{10 a} \cdot T^{10 a}\right)_{2 c}^{2 b}\left(T_{10 a} \cdot T^{10 a}\right)_{2 b}^{2 c}+\tau\left(T_{9 a} \cdot T^{9 a}\right)_{3 c}^{3 b}\left(T_{9 a} \cdot T^{9 a}\right)_{3 b}^{3 c} \\
& +\nu\left(T_{8 a} \cdot T^{8 a}\right)_{4 c}^{4 b}\left(T_{8 a} \cdot T^{8 a}\right)_{4 b}^{4 c}+\sigma\left(T_{7 a} \cdot T^{7 a}\right)_{5 c}^{5 b}\left(T_{7 a} \cdot T^{7 a}\right)_{5 b}^{5 c} \\
& +\chi\left(T_{6 a} \cdot T^{6 a}\right)_{6 c}^{6 b}\left(T_{6 a} \cdot T^{6 a}\right)_{6 b}^{6 c} .
\end{aligned}
$$

This notation is consistent when the tensor $T$ is totally symmetric on all of its indices. ${ }^{2}$

\section{$2.4 \mathrm{SU}(3)$ potentials}

Similar to the previous section, the omission of cubic terms means that the following potentials have an $\mathrm{SU}(3) \times \mathrm{U}(1)$ symmetry, where the $\mathrm{U}(1)$ can be dealt with as described above.

\footnotetext{
${ }^{2}$ We could write an even more compact notation in generalized dyadic form, e.g., the $\nu$ term would be $\nu\left(T:^{8} T\right):^{4}\left(T:^{8} T\right)$ which again defines how the tensor contractions are to be made, but we find this form unnecessary here. However, it could be useful for expressions involving more complicated group invariants. Cvitanovic's "Bird Track" notation [29] can also be useful for this purpose.
} 


\subsection{1 $\quad A_{4}$ and $T_{7}$}

In addition to $\mathrm{SO}(3), A_{4}$ can originate from a broken $\mathrm{SU}(3)$ symmetry. Looking at table 24 we see that the lowest dimensional irrep containing a trivial $A_{4}$ singlet is the $\mathbf{6}$, but as explained in [19], neither the $\mathbf{6}, \mathbf{1 0}$, nor $\mathbf{1 5}^{\prime}$ will break $\mathrm{SU}(3)$ uniquely to $A_{4}$, i.e., giving these irreps an $A_{4} \mathrm{VEV}$ will necessarily leave a group larger than $A_{4}$ unbroken. This leaves us with the $\mathbf{1 5}$ as the smallest irrep that will uniquely break to an $A_{4}$ subgroup, and the same logic applies to $T_{7}$. (A variety of $T_{7}$ models have been proposed, see [47-50].) To obtain a useful form of the $\mathbf{1 5}$ we first take the product $\mathbf{3} \times \mathbf{3} \times \overline{\mathbf{3}}$ in $\mathrm{SU}(3)$; then by specifying the part that is symmetric on 2 indices, $S_{i j}^{k}$, we reduce the number of independent components from 27 to 18 . Finally, subtracting off the three traces: $\sum_{j}^{3} \delta_{j k} S_{i j}^{k}, i=1,2,3$, gives us the desired 15 component tensor. The associated potential [19] is

$$
\begin{aligned}
V_{15}= & -m^{2} T_{i j}^{k} T_{k}^{i j}+\lambda\left(T_{i j}^{k} T_{k}^{i j}\right)^{2}+\kappa T_{j m}^{i} T_{i}^{j n} T_{l n}^{k} T_{k}^{l m} \\
& +\rho T_{j m}^{i} T_{i}^{j n} T_{k l}^{m} T_{n}^{k l}+\tau T_{i j}^{m} T_{n}^{i j} T_{k l}^{n} T_{m}^{k l}+\nu T_{j m}^{i} T_{i n}^{j} T_{l}^{k m} T_{k}^{l n} .
\end{aligned}
$$

\section{$2.4 .2 \Delta(27)$}

From table 26 we see that we can use the $\mathbf{1 0}$ to spontaneously break from SU(3) to $\Delta(27)$. We can get to this irrep by taking the product of three triplets and specifying the fully symmetric part of the resulting tensor, which reduces to the desired ten independent components. The potential is

$$
V_{10}=-m^{2} T_{i j k} T^{i j k}+\lambda\left(T_{i j k} T^{i j k}\right)^{2}+\kappa T_{i j m} T^{i j n} T_{k l n} T^{k l m} .
$$

where the cubic terms have vanished upon summation. This result can also be found in [19]. Examples where $\Delta(27)$ has been used to build models can be found in [51, 52].

\subsubsection{PSL $(2,7)$}

Another group that has garnered considerable interest as a flavor symmetry is $\operatorname{PSL}(2,7)$ [53]. Looking at table 27 we see that the lowest dimensional irrep of SU(3) we can use to break to $\operatorname{PSL}(2,7)$ is the $\mathbf{1 5}^{\prime}$, (Dynkin label $\left[\begin{array}{ll}4 & 0\end{array}\right]$ ). To get to a $\mathbf{1 5}^{\prime}$ we take the product of four fundamental triplets

$$
\mathbf{3} \times \mathbf{3} \times \mathbf{3} \times \mathbf{3}=3 \cdot \mathbf{3}+2 \cdot \overline{\mathbf{6}}+3 \cdot \mathbf{1 5}+\mathbf{1 5 ^ { \prime }} .
$$

The generic rank 4 tensor has 81 independent components, requiring it be symmetric on all four indices reduces it to $\mathbf{1 5}^{\prime}$ as required. The associated potential is

$$
\begin{aligned}
V_{15^{\prime}}= & -m^{2} T_{i j k l} T^{i j k l}+\lambda\left(T_{i j k l} T^{i j k l}\right)^{2} \\
& +\kappa T_{i j k l} T^{i j k m} T_{m n o p} T^{l n o p}+\rho T_{i j k l} T^{i j m n} T_{m n o p} T^{k l o p} .
\end{aligned}
$$

Also of interest is the next lowest irrep suitable for breaking from $\operatorname{SU}(3)$ to $\operatorname{PSL}(2,7)$, the 28. We build this irrep by taking the symmetric product of six triplets, giving a fully symmetric rank 6 tensor with 28 components. The associated potential is

$$
\begin{aligned}
V_{28}= & -m^{2} T_{i j k l m n} T^{i j k l m n}+\lambda\left(T_{i j k l m n} T^{i j k l m n}\right)^{2} \\
& +\kappa T_{i j k l m n} T^{i j k l m t} T_{\text {opqrst }} T^{\text {opqrsn }}+\rho T_{i j k l m n} T^{i j k l s t} T_{\text {opqrst }} T^{\text {opqrmn }} \\
& +\tau T_{i j k l m n} T^{i j k r s t} T_{\text {opqrst }} T^{\text {opqlmn }} .
\end{aligned}
$$




\section{$3 \quad$ Vaccuum alignments for spontaneous symmetry breaking}

\subsection{Vacuua for $\mathrm{SO}(3)$ potentials}

The invariant tensors from the previous section can be written in terms of a $d$-dimensional orthonormal basis, where $d$ is the number of independent tensor components. To illustrate this consider the $\mathbf{5}$ of $\mathrm{SO}(3)$ which is a second rank symmetric traceless tensor, $T_{i j}$. It has a basis

$$
\begin{aligned}
|1\rangle & =\frac{1}{\sqrt{2}}(|11\rangle-|22\rangle) ; \\
|2\rangle & =\frac{1}{\sqrt{6}}(|11\rangle+|22\rangle-2 \cdot|33\rangle) ; \\
|3\rangle & =\frac{1}{\sqrt{2}}(|12\rangle+|21\rangle) ; \\
|4\rangle & =\frac{1}{\sqrt{2}}(|13\rangle+|31\rangle) ; \\
|5\rangle & =\frac{1}{\sqrt{2}}(|23\rangle+|32\rangle) .
\end{aligned}
$$

Where $|i j\rangle$ is the $i j^{\text {th }}$ component of the tensor. Using this basis the matrix form of $T_{i j}$ is

$$
T_{i j}=\left(\begin{array}{ccc}
\frac{1}{\sqrt{2}}|1\rangle+\frac{1}{\sqrt{6}}|2\rangle & \frac{1}{\sqrt{2}}|3\rangle & \frac{1}{\sqrt{2}}|4\rangle \\
\frac{1}{\sqrt{2}}|3\rangle & -\frac{1}{\sqrt{2}}|1\rangle+\frac{1}{\sqrt{6}}|2\rangle & \frac{1}{\sqrt{2}}|5\rangle \\
\frac{1}{\sqrt{2}}|4\rangle & \frac{1}{\sqrt{2}}|5\rangle & -\sqrt{\frac{2}{3}}|2\rangle
\end{array}\right) .
$$

With an explicit basis, it now makes sense to look for a d-component vacuum alignment that minimizes the potential and is invariant under the desired subgroup. How do we find this specified direction? First, note that we can express our basis above in polynomial form, assigning component 1 to $x, 2$ to $y$, and 3 to $z$ :

$$
\begin{aligned}
& |1\rangle=\frac{1}{\sqrt{2}}\left(x^{2}-y^{2}\right) ; \\
& |2\rangle=\frac{1}{\sqrt{6}}\left(x^{2}+y^{2}-2 z^{2}\right) ; \\
& |3\rangle=\frac{1}{\sqrt{2}}(x y+y x)=\sqrt{2} x y ; \\
& |4\rangle=\sqrt{2} x z ; \\
& |5\rangle=\sqrt{2} y z .
\end{aligned}
$$

So if we find a polynomial that is invariant under the desired subgroup we can convert it into a vacuum alignment by expressing it as a vector in terms of these basis functions [20]. To find a polynomial, $\mathcal{I}(x, y, z)$, invariant under a group $H$, one employs the Reynolds Operator $[16,17]$

$$
\mathcal{I}(x, y, z)=\frac{1}{|\mathcal{R}(H)|} \sum_{h \in \mathcal{R}(H)} f\left(h \circ\left(\begin{array}{l}
x \\
y \\
z
\end{array}\right)\right) .
$$


Where $\mathcal{R}(H)$ is a representation of the group, $|\mathcal{R}(H)|$ is the number of elements in the group, and $f\left(h \circ\left(\begin{array}{l}x \\ y \\ z\end{array}\right)\right)$ signifies the result of a group element $h$ acting on the vector $(x, y, z)$ and then input into a trial function $f(x, y, z)$. Taking a group representation and a trial function as inputs, it will output a polynomial invariant of the group. Often this output will be zero, the trivial invariant, but with enough educated guesses for the trial functions, a non-trivial invariant is easily obtained in most cases. Trial polynomials of the form $x^{n} y^{m} z^{d-n-m}$ will typically be most useful in finding invariants of degree $d$. Note we have specified polynomials in three variables here, but we can use the same procedure to find invariants in terms of any number of variables, real or complex. E.g., in two real dimensions we can find an invariant $\mathcal{I}(x, y)$ with a trial function $f(x, y)$.

Many of the invariants we use have been obtained by Merle and Zwicky and are available in their Mathematica package SUTree. The invariants for the $\mathrm{SU}(2)$ cases we needed were not in SUTree, but were generated using their Reynolds Operator methods [20].

\subsection{1 $\quad A_{4}$}

As an initial practical example lets examine the symmetry breaking pattern $\mathrm{SO}(3) \rightarrow A_{4}$. The irrep of interest is a $\mathbf{7}$ which is the symmetric, traceless part of $\mathbf{3} \times \mathbf{3} \times \mathbf{3}$. Expressed it in terms of 7 orthonormal components we have

$$
\begin{aligned}
&|1\rangle=\frac{1}{2}(|111\rangle-|122\rangle-|212\rangle-|221\rangle), \\
&|2\rangle=\frac{1}{\sqrt{60}}(3 \cdot|111\rangle+|122\rangle+|212\rangle+|221\rangle-4 \cdot|133\rangle-4 \cdot|313\rangle-4 \cdot|331\rangle), \\
&|3\rangle=\frac{1}{2}(|222\rangle-|112\rangle-|121\rangle-|211\rangle), \\
&|4\rangle=\frac{1}{\sqrt{60}}(3 \cdot|222\rangle+|112\rangle+|121\rangle+|211\rangle-4 \cdot|233\rangle-4 \cdot|323\rangle-4 \cdot|332\rangle), \\
&|5\rangle=\frac{1}{2}(|333\rangle-|113\rangle-|131\rangle-|311\rangle), \\
&|6\rangle=\frac{1}{\sqrt{60}}(3 \cdot|333\rangle+|113\rangle+|131\rangle+|311\rangle-4 \cdot|223\rangle-4 \cdot|232\rangle-4 \cdot|322\rangle), \\
&|7\rangle=\frac{1}{\sqrt{6}}(|123\rangle+|132\rangle+|213\rangle+|231\rangle+|312\rangle+|321\rangle) .
\end{aligned}
$$

Using $x y z$ as a trial polynomial in equation $(3.3),(d=3, n=m=1)$ gives us back $x y z$ as our invariant polynomial. Expressed in terms of this basis our $A_{4}$ invariant vacuum alignment is remarkably simple:

$$
v=[0,0,0,0,0,0,1]
$$

The VEV for spontaneous breaking will be this unit vector multiplied by a constant which minimizes the potential. Next we must show that this VEV is unique to $A_{4}$. The gauge group will spontaneously break to the largest subgroup which leaves that VEV invariant. So $G$ will only break to a desired subgroup, $H$, if there is no other group, $H^{\prime}$, which is invariant under the specified VEV and satisfies $H \subset H^{\prime} \subset G$. Unless $H$ is maximal 
in $G$, it is difficult to systematically determine which subgroup will be left invariant for a given breaking, so each case must be considered individually. For the present case we start with the fact that the only groups that contain $A_{4}$ and are subgroups of $\mathrm{SO}(3)$ are $S_{4}$ and $A_{5}$. Examining the branching rules for both these groups, one sees that a $\mathbf{7}$ of $\mathrm{SO}(3)$ does not break to a trivial singlet of either $S_{4}$ or $A_{5}$, and thus the largest group left invariant by this VEV must be $A_{4}$. Hence we have obtained the desired result for the case at hand.

Let us pause to expand on the above remarks. Note that we can not have an unbroken continuous group in addition to $A_{4}$, since there is just not enough room in $\mathrm{SO}(3)$ to allow it. The only continuous subgroups of $\mathrm{SO}(3)$ are $\mathrm{SO}(2)$ and $\mathrm{U}(1)$ and they are both abelian. If we think of $A_{4}$ as the rotation group of the tetrahedron, then we need finite linear combinations of all the $\mathrm{SO}(3)$ to do these rotations, indicating that there are no remaining $\mathrm{SO}(3)$ to identify with an additional factor in a subgroup like $A_{4} \times \mathrm{U}(1)$. We can generalize the previous statement to apply to the binary tetrahedral and binary icosahedral groups of $\mathrm{SU}(2)$. Similar logic applies to several SU(3) cases while for others we refer to the literature. We will comment on various individual cases below.

\section{$3.1 .2 \quad S_{4}$}

For the $\mathbf{9}$ of $\mathrm{SO}(3)$, it is more convenient, and yields equivalent results (up to a normalization $)^{3}$ to express our basis in terms of spherical harmonics of degree $1=4, Y_{4}^{m}$ (where $m=$ $-4,-3 \ldots 0 \ldots 3,4)$. In order to get real basis vectors, we define them as:

$$
\begin{aligned}
& |1\rangle=Y_{4}^{0} ; \quad|2\rangle=\frac{i}{\sqrt{2}}\left(Y_{4}^{1}+Y_{4}^{-1}\right) ; \\
& |3\rangle=\frac{1}{\sqrt{2}}\left(Y_{4}^{1}-Y_{4}^{-1}\right) ; \quad|4\rangle=\frac{1}{\sqrt{2}}\left(Y_{4}^{2}+Y_{4}^{-2}\right) ; \\
& |5\rangle=\frac{i}{\sqrt{2}}\left(Y_{4}^{2}-Y_{4}^{-2}\right) ; \quad|6\rangle=\frac{i}{\sqrt{2}}\left(Y_{4}^{3}+Y_{4}^{-3}\right) ; \quad|7\rangle=\frac{1}{\sqrt{2}}\left(Y_{4}^{3}-Y_{4}^{-3}\right) ; \\
& |8\rangle=\frac{1}{\sqrt{2}}\left(Y_{4}^{4}+Y_{4}^{-4}\right) ; \quad|9\rangle=\frac{i}{\sqrt{2}}\left(Y_{4}^{4}-Y_{4}^{-4}\right) .
\end{aligned}
$$

We find that the polynomial, $x^{4}+y^{4}+z^{4}$ is $S_{4}$ invariant. Expressed in terms of our basis this is

$$
v=\left[\sqrt{\frac{7}{5}}, 0,0,0,0,0,0,1,0\right] .
$$

Because $S_{4}$ is a maximal subgroup of $\mathrm{SO}(3)$, i.e., there is no group $H^{\prime}$ that nontrivially satisfies $S_{4} \subset H^{\prime} \subset \mathrm{SO}(3)$, so for any VEV of the $\mathbf{9}$, we can be sure the VEV in eq. (3.7) breaks $\mathrm{SO}(3)$ uniquely to $S_{4}$.

\subsection{3 $\quad A_{5}$}

As mentioned previously, to break from $\mathrm{SO}(3)$ to $A_{5}$ the irrep of interest is the totally symmetric traceless tensor with 13 independent components contained in $\mathbf{3} \times \mathbf{3} \times \mathbf{3} \times \mathbf{3} \times \mathbf{3} \times \mathbf{3}$. In this case it is again easier to express the components in terms

\footnotetext{
${ }^{3}$ One can also use this method for the $A_{4}$ case, see [54].
} 
of spherical harmonics, in this case of degree $\mathrm{l}=6, Y_{6}^{m}$ (where $\left.m=-6,-5 \ldots 0 \ldots 5,6\right)$. In order to get real basis vectors, we define them as

$$
\begin{aligned}
& |1\rangle=Y_{6}^{0} ; \quad|2\rangle=\frac{i}{\sqrt{2}}\left(Y_{6}^{1}+Y_{6}^{-1}\right) ; \\
& |3\rangle=\frac{1}{\sqrt{2}}\left(Y_{6}^{1}-Y_{6}^{-1}\right) ; \quad|4\rangle=\frac{1}{\sqrt{2}}\left(Y_{6}^{2}+Y_{6}^{-2}\right) ; \\
& |5\rangle=\frac{i}{\sqrt{2}}\left(Y_{6}^{2}-Y_{6}^{-2}\right) ; \quad|6\rangle=\frac{i}{\sqrt{2}}\left(Y_{6}^{3}+Y_{6}^{-3}\right) ; \quad|7\rangle=\frac{1}{\sqrt{2}}\left(Y_{6}^{3}-Y_{6}^{-3}\right) ; \\
& |8\rangle=\frac{1}{\sqrt{2}}\left(Y_{6}^{4}+Y_{6}^{-4}\right) ; \quad|9\rangle=\frac{i}{\sqrt{2}}\left(Y_{6}^{4}-Y_{6}^{-4}\right) ; \quad|10\rangle=\frac{i}{\sqrt{2}}\left(Y_{6}^{5}+Y_{6}^{-5}\right) ; \\
& |11\rangle=\frac{1}{\sqrt{2}}\left(Y_{6}^{5}-Y_{6}^{-5}\right) ; \quad|12\rangle=\frac{1}{\sqrt{2}}\left(Y_{6}^{6}+Y_{6}^{-6}\right) ; \quad|13\rangle=\frac{i}{\sqrt{2}}\left(Y_{6}^{6}-Y_{6}^{-6}\right) \text {. }
\end{aligned}
$$

We find that a degree six invariant polynomial is $\left(\frac{(1+\sqrt{5})^{2}}{4} x^{2}-y^{2}\right)\left(\frac{(1+\sqrt{5})^{2}}{4} y^{2}-\right.$ $\left.z^{2}\right)\left(\frac{(1+\sqrt{5})^{2}}{4} z^{2}-x^{2}\right)[20]$. The associated VEV is proportional to

$$
v=\left[1,0,0,-\sqrt{\frac{21}{2}}, 0,0,0,-\sqrt{7}, 0,0,0, \sqrt{\frac{105}{22}}, 0\right] .
$$

As in the case of $S_{4}, A_{5}$ is also a maximal subgroup of $\mathrm{SO}(3)$, so we can be certain our alignment breaks $\mathrm{SO}(3)$ uniquely to $A_{5}$.

\subsection{Vacuua for $\mathrm{SU}(2)$ potentials}

\subsection{1 $T^{\prime}$}

Because $\mathrm{SU}(2)$ breaks to $T^{\prime}$ from the same real seven dimensional irrep that breaks $\mathrm{SO}(3)$ to $A_{4}$, the potentials are the same and the basis will be the same as in the $A_{4}$ section above. In addition, the Reynolds operator yields the same polynomial invariant $x y z$, so the VEV is identical. On the other hand the complex $\mathbf{7}$ has a different origin, specifically that of the symmetric tensor with 6 indices: ${ }^{4}$

$$
\begin{aligned}
& |1\rangle=|111111\rangle ; \\
& \begin{aligned}
&|2\rangle= \frac{1}{\sqrt{6}}(|111112\rangle+|111121\rangle+|111211\rangle+|112111\rangle+|121111\rangle+|211111\rangle) ; \\
&|3\rangle=\frac{1}{\sqrt{15}}(|111122\rangle+|111212\rangle+|111221\rangle+|112112\rangle+|112121\rangle+|112211\rangle \\
& \quad+|121112\rangle+|121121\rangle+|121211\rangle+|122111\rangle+|211112\rangle \\
&\quad+|211121\rangle+|211211\rangle+|212111\rangle+|221111\rangle) ; \\
& \quad+|121221\rangle+|122112\rangle+|122121\rangle+|122211\rangle+|211122\rangle
\end{aligned} \\
& \begin{array}{r}
|4\rangle=\frac{1}{\sqrt{20}}(|11122\rangle+|112122\rangle+|112212\rangle+|112221\rangle+|121122\rangle+|121212\rangle \\
\quad
\end{array}
\end{aligned}
$$

\footnotetext{
${ }^{4}$ Because this is a complex irrep there are actually 14 basis states; the basis states listed are the 7 real parts of the tensor components, while bases 8 through 14 are the imaginary parts. These conjugate components have been suppressed here since they will always be set to zero at vacuum in order to have a real VEV. This will be the case for all of the complex irreps we consider.
} 


$$
\begin{gathered}
+|211212\rangle+|211221\rangle+|212112\rangle+|212121\rangle+|212211\rangle \\
+|221112\rangle+|221121\rangle+|221211\rangle+|222111\rangle) \\
|5\rangle=\frac{1}{\sqrt{15}}(|112222\rangle+|121222\rangle+|122122\rangle+|122212\rangle+|122221\rangle+|211222\rangle \\
+|212122\rangle+|212212\rangle+|212221\rangle+|221122\rangle+|221212\rangle \\
+|221221\rangle+|222112\rangle+|222121\rangle+|222211\rangle \\
|6\rangle=\frac{1}{\sqrt{6}}(|122222\rangle+|212222\rangle+|221222\rangle+|222122\rangle+|222212\rangle+|222221\rangle \\
|7\rangle=|22222\rangle
\end{gathered}
$$

We find that the polynomial $\frac{1}{2}\left(x y^{5}-y x^{5}\right)$ is left invariant by $T^{\prime}$ for this representation and the associated VEV is proportional to

$$
v=[0,-1,0,0,0,1,0 ; 0,0,0,0,0,0,0] .
$$

(Where we have divided the real and imaginary components of the basis with a ";"). To make sure we have broken to $\mathrm{T}^{\prime}$ we must show that this VEV does not break $\mathrm{SU}(2)$ to any larger group. The only $\mathrm{SU}(2)$ subgroups that contain $\mathrm{T}^{\prime}$ as a subgroup are $\mathrm{I}^{\prime}$, the binary icosahedral group, and $O^{\prime}$, the binary octahedral group. Looking at tables of branching rules we see that the $\mathbf{7}$ of SU(2) does not contain a trivial singlet of either of these groups, so we can be certain the breaking is to $T^{\prime}$ as desired.

\section{$3.2 .2 \quad Q_{6}$}

For the breaking $\mathrm{SU}(2) \rightarrow Q_{6}$ we use the same basis for the complex $\mathbf{7}$ as with $T^{\prime}$ above. We find the polynomial $\frac{1}{2}\left(x^{6}+y^{6}\right)$ is left invariant by $Q_{6}$, and this leads to a VEV proportional to

$$
v=[1,1,0,0,0,0,0 ; 0,0,0,0,0,0,0] .
$$

To make sure we have broken to $Q_{6}$ and not any larger subgroups, we first note that the 7 does not break to any $Q_{n}$ with $n>6$ (see page 6 of [37]). The only other larger $\mathrm{SU}(2)$ subgroup that can be spontaneously broken with a $\mathbf{7}$ is $T^{\prime}$, but we find that $T^{\prime}$ has only one degree six invariant which is given in the subsection above. Therefore, the VEV in eq. (3.12) is the result we were seeking.

\subsection{3 $O^{\prime}$}

Like the other double cover groups, the basis and vacuum direction for the breaking of $O^{\prime}$ with a real 9 of $\mathrm{SU}(2)$ will be the same as its $\mathrm{SO}(3) \rightarrow S_{4}$ counterpart above.

The complex $\mathbf{9}$ arises from the basis of the symmetric tensor with 8 doublet indices:

$$
\begin{array}{rlrl}
|1\rangle & =|11111111\rangle ; & |2\rangle=|22222222\rangle ; \\
|3\rangle=\frac{1}{\sqrt{8}}(|11111112\rangle+\text { perms }) ; & & \\
|4\rangle=\frac{1}{\sqrt{8}}(|22222221\rangle+\text { perms }) ; & |5\rangle=\frac{1}{\sqrt{28}}(|11111122\rangle+\text { perms }) ; \\
|6\rangle=\frac{1}{\sqrt{28}}(|22222211\rangle+\text { perms }) ; & |7\rangle=\frac{1}{\sqrt{56}}(|11111222\rangle+\text { perms }) ; \\
|8\rangle=\frac{1}{\sqrt{56}}(|22222111\rangle+\text { perms }) ; & |9\rangle=\frac{1}{\sqrt{70}}(|11112222\rangle+\text { perms }),
\end{array}
$$


where here and in what follows '+perms' means we include all inequivalent permutations of tensor indices.

Here the relevant $O^{\prime}$ invariant polynomial is $x^{8}+y^{8}+14 x^{4} y^{4}$, which leads to a VEV proportional to

$$
v=\left[1,1,0,0,0,0,0,0, \frac{14}{\sqrt{70}} ; 0,0,0,0,0,0,0,0,0\right] .
$$

Where only $|1\rangle=|2\rangle$ and $|9\rangle=\frac{14}{\sqrt{70}}|1\rangle$ are nonvanishing. $O^{\prime}$ is a maximal subgroup of $\mathrm{SU}(2)$, so we can be certain our alignment breaks $\mathrm{SU}(2)$ uniquely to $O^{\prime}$.

\subsection{4 $\quad I^{\prime}$}

Similar to the spontaneous symmetry breaking behavior of the $T^{\prime}$ case relative to the $A_{4}$ case with a real $\mathbf{7}$, the basis for the symmetry breaking to $I^{\prime}$ with the real $\mathbf{1 3}$ will be the same as for $A_{5}$ above. Additionally, both groups have the same invariant polynomial so the vacuum directions will be the same.

On the other hand, a complex $\mathbf{1 3}$ arises from the basis of the symmetric tensor with 12 doublet indices:

$$
\begin{aligned}
|1\rangle & =|111111111111\rangle ; & |2\rangle & =|222222222222\rangle ; \\
|3\rangle & =\frac{1}{\sqrt{12}}(|111111111112\rangle+\text { perms }) ; & & \\
|4\rangle & =\frac{1}{\sqrt{12}}(|222222222221\rangle+\text { perms }) ; & |5\rangle & =\frac{1}{\sqrt{66}}(|111111111122\rangle+\text { perms }) ; \\
|6\rangle & =\frac{1}{\sqrt{66}}(|222222222211\rangle+\text { perms }) ; & |7\rangle & =\frac{1}{\sqrt{220}}(|111111111222\rangle+\text { perms }) ; \\
|8\rangle & =\frac{1}{\sqrt{220}}(|222222222111\rangle+\text { perms }) ; & |9\rangle & =\frac{1}{\sqrt{495}}(|1111111112222\rangle+\text { perms }) ; \\
|10\rangle & =\frac{1}{\sqrt{495}}(|222222221111\rangle+\text { perms }) ; & |11\rangle & =\frac{1}{\sqrt{792}}(|111111122222\rangle+\text { perms }) ; \\
|12\rangle & =\frac{1}{\sqrt{792}}(|222222211111\rangle+\text { perms }) ; & |13\rangle & =\frac{1}{\sqrt{924}}(|222222111111\rangle+\text { perms }) .
\end{aligned}
$$

Here the $I^{\prime}$ invariant polynomial is $x^{11} y+11 x^{6} y^{6}-y^{11} x$, which leads to a VEV proportional to

$$
v=\left[0,0,1,-1,0,0,0,0,0,0,0,0, \sqrt{\frac{11}{12}} ; 0,0,0,0,0,0,0,0,0,0,0,0,0\right],
$$

Where only $|4\rangle=-|3\rangle$ and $|13\rangle=\sqrt{\frac{11}{12}} \cdot|3\rangle$ are nonvanishing. $I^{\prime}$ is a known maximal subgroup of $\mathrm{SU}(2)$, so we can be certain our alignment breaks $\mathrm{SU}(2)$ uniquely to $I^{\prime}$.

\subsection{Vacuua for $\mathrm{SU}(3)$ potentials}

First let us show that we can get nonmaximal discrete subgroups from continuous groups. For this purpose we use the example $\mathrm{SU}(3) \rightarrow A_{4}$ where we break with a $\mathbf{1 5}$ of $\mathrm{SU}(3)$. Then we find vacuua for the maximal cases discussed above. Then finally, for $\operatorname{PSL}(2,7)$ we give both a minimal case with a VEV for the $\mathbf{1 5}^{\prime}$ of $\mathrm{SU}(3)$ and a nonminimal breaking via a $\mathbf{2 8}$ of $\mathrm{SU}(3)$ using the potential given in eq. (2.15). 


\subsection{1 $\quad A_{4}$}

The complex 15 dimensional basis needed to break $\mathrm{SU}(3)$ to $A_{4}$ is that of the traceless $3 \times 3 \times \overline{3}$ tensor that is symmetric on the first two indices [19]. For a basis we write

$$
\begin{aligned}
|1\rangle & =\frac{1}{\sqrt{3}}(|111\rangle-|122\rangle-|212\rangle) ; \\
|2\rangle & =\frac{1}{2 \sqrt{6}}(2 \cdot|111\rangle+|122\rangle+|212\rangle-3 \cdot|133\rangle-3 \cdot|313\rangle) ; \\
|3\rangle & =\frac{1}{\sqrt{3}}(|222\rangle-|233\rangle-|323\rangle) ; \\
|4\rangle & =\frac{1}{2 \sqrt{6}}(2 \cdot|222\rangle+|233\rangle+|323\rangle-3 \cdot|211\rangle-3 \cdot|121\rangle) ; \\
|5\rangle & =\frac{1}{\sqrt{3}}(|333\rangle-|311\rangle-|131\rangle) ; \\
|6\rangle & =\frac{1}{2 \sqrt{6}}(2 \cdot|333\rangle+|311\rangle+|131\rangle-3 \cdot|322\rangle-3 \cdot|232\rangle) ; \\
|7\rangle & =|112\rangle ; \quad|8\rangle=|113\rangle ; \quad|11\rangle=|331\rangle ; \quad|12\rangle=|332\rangle ; \\
|10\rangle & =|221\rangle ; \quad|14\rangle=\frac{1}{\sqrt{2}}(|231\rangle+|321\rangle) ; \quad|15\rangle=\frac{1}{\sqrt{2}}(|312\rangle+|132\rangle) \\
|13\rangle & =\frac{1}{\sqrt{2}}(|123\rangle+|213\rangle) ; \quad|323\rangle ;
\end{aligned}
$$

Because this tensor is constructed from the product $3 \times 3 \times \overline{3}$, the invariant should be of degree 2 in the variables $x, y, z$ and degree 1 in the conjugate variables, $x^{*}, y^{*}, z^{*}$. Inputting the trial polynomial $x y z^{*}$ into the Reynolds operator produces the invariant: $x y z^{*}+y z x^{*}+x z y^{*}$. In the basis of eq. (3.17) the VEV is proportional to

$$
v=[0,0,0,0,0,0,0,0,0,0,0,0,1,1,1 ; 0,0,0,0,0,0,0,0,0,0,0,0,0,0,0]
$$

i.e., where only $|13\rangle=|14\rangle=|15\rangle$ are nonzero.

One can examine the generators of $A_{4}$ and SU(3) to see that this VEV breaks SU(3) uniquely to $A_{4}$, see [19].

\subsection{2 $\quad T_{7}$}

The irrep we use and also the basis we need for $T_{7}$ are the same as for $A_{4}$ above. The invariant polynomial in this case is $x^{2} y^{*}+y^{2} z^{*}+z^{2} x^{*}$ and the corresponding VEV is proportional to

$$
v=[0,0,0,0,0,0,1,0,1,0,1,0,0,0,0 ; 0,0,0,0,0,0,0,0,0,0,0,0,0,0,0]
$$

where $|7\rangle=|9\rangle=|11\rangle$ are nonvanishing.

Similar to the $A_{4}$ case, one can verify this VEV uniquely breaks $\mathrm{SU}(3)$ to $T_{7}$ by examining how the $T_{7}$ generators operate on $v$, see [19]. 


\subsection{3 $\Delta(27)$}

For $\Delta(27)$, the relevant invariant tensor is the fully symmetric part of $3 \times 3 \times 3$ with 10 independent components

$$
\begin{aligned}
& |1\rangle=|111\rangle ;|2\rangle=|222\rangle ;|3\rangle=|333\rangle ; \\
& |4\rangle=\frac{1}{\sqrt{3}}(|112\rangle+|121\rangle+|211\rangle) ; \quad|5\rangle=\frac{1}{\sqrt{3}}(|113\rangle+|131\rangle+|311\rangle) ; \\
& |6\rangle=\frac{1}{\sqrt{3}}(|221\rangle+|212\rangle+|122\rangle) ;|7\rangle=\frac{1}{\sqrt{3}}(|223\rangle+|232\rangle+|322\rangle) ; \\
& |8\rangle=\frac{1}{\sqrt{3}}(|331\rangle+|313\rangle+|133\rangle) ;|9\rangle=\frac{1}{\sqrt{3}}(|332\rangle+|323\rangle+|233\rangle) ; \\
& |10\rangle=\frac{1}{\sqrt{6}}(|123\rangle+|231\rangle+|312\rangle+|321\rangle+|213\rangle+|132\rangle) .
\end{aligned}
$$

The $\Delta(27)$ invariant polynomial is $x^{3}+y^{3}+z^{3}$, which leads to a VEV proportional to

$$
v=[1,1,1,0,0,0,0,0,0,0 ; 0,0,0,0,0,0,0,0,0,0]
$$

Again explicit forms of the generators can be examined in order to verify the uniqueness of this VEV for breaking from $\mathrm{SU}(3)$ to $\Delta(27)$ [19].

\subsubsection{PSL $(2,7)$}

Our basis for the $\mathbf{1 5}^{\prime}$ comes from the fully symmetric $3 \times 3 \times 3 \times 3$ tensor and is

$$
\begin{aligned}
|1\rangle & =|1111\rangle ; & |2\rangle & =|2222\rangle ; \\
|3\rangle & =|3333\rangle ; & & \\
|4\rangle & =\frac{1}{2}(|1112\rangle+|1121\rangle+|1211\rangle+|2111\rangle) ; & |5\rangle & =\frac{1}{2}(|1113\rangle+|1131\rangle+|1311\rangle+|3111\rangle) ; \\
|6\rangle & =\frac{1}{2}(|2221\rangle+|2212\rangle+|2122\rangle+|1222\rangle) ; & |7\rangle & =\frac{1}{2}(|2223\rangle+|2232\rangle+|2322\rangle+|3222\rangle) ; \\
|8\rangle & =\frac{1}{2}(|3331\rangle+|3313\rangle+|3133\rangle+|1333\rangle) ; & |9\rangle & =\frac{1}{2}(|3332\rangle+|3323\rangle+|3233\rangle+|2333\rangle) ; \\
|10\rangle & =\frac{1}{\sqrt{6}}(|1122\rangle+\text { perms }) ; & |11\rangle & =\frac{1}{\sqrt{6}}(|1133\rangle+\text { perms }) ; \\
|12\rangle & =\frac{1}{\sqrt{6}}(|2233\rangle+\text { perms }) ; & & \\
|13\rangle & =\frac{1}{\sqrt{12}}(|1123\rangle+\text { perms }) ; & & \\
|15\rangle & =\frac{1}{\sqrt{12}}(|3312\rangle+\text { perms }) . & &
\end{aligned}
$$

The relevant $\operatorname{PSL}(2,7)$ invariant polynomial is $x^{3} z+y^{3} x+z^{3} y$ [20], which gives a VEV proportional to

$$
v=[0,0,0,0,1,1,0,0,1,0,0,0,0,0,0 ; 0,0,0,0,0,0,0,0,0,0,0,0,0,0,0],
$$


where the nonvanishing vacuum components are $|5\rangle=|6\rangle=|9\rangle$. We can be sure we have broken to the correct $\operatorname{subgroup}^{5}$ because $\operatorname{PSL}(2,7)$ is known to be a maximal in $\operatorname{SU}(3)$.

Finally, for the $\mathbf{2 8}$ of $\mathrm{SU}(3)$ we write the basis for the fully symmetric $\mathbf{3}^{6}$ tensor in the form

$$
\begin{aligned}
|1\rangle & =|111111\rangle ; & |2\rangle & =|22222\rangle ; \\
|3\rangle & =|333333\rangle ; & |4\rangle & =\frac{1}{\sqrt{6}}(|111112\rangle+\text { perms }) ; \\
|5\rangle & =\frac{1}{\sqrt{6}}(|111113\rangle+\text { perms }) ; & |6\rangle & =\frac{1}{\sqrt{6}}(|222221\rangle+\text { perms }) ; \\
|7\rangle & =\frac{1}{\sqrt{6}}(|222223\rangle+\text { perms }) ; & |8\rangle & =\frac{1}{\sqrt{6}}(|333331\rangle+\text { perms }) ; \\
|9\rangle & =\frac{1}{\sqrt{6}}(|333332\rangle+\text { perms }) ; & |10\rangle & =\frac{1}{\sqrt{15}}(|111122\rangle+\text { perms }) ; \\
|11\rangle & =\frac{1}{\sqrt{15}}(|111133\rangle+\text { perms }) ; & |12\rangle & =\frac{1}{\sqrt{15}}(|222211\rangle+\text { perms }) ; \\
|13\rangle & =\frac{1}{\sqrt{15}}(|222233\rangle+\text { perms }) ; & |14\rangle & =\frac{1}{\sqrt{15}}(|333311\rangle+\text { perms }) ; \\
|15\rangle & =\frac{1}{\sqrt{15}}(|333322\rangle+\text { perms }) ; & |16\rangle & =\frac{1}{\sqrt{30}}(|111123\rangle+\text { perms }) ; \\
|17\rangle & =\frac{1}{\sqrt{30}}(|222231\rangle+\text { perms }) ; & |18\rangle & =\frac{1}{\sqrt{30}}(|333312\rangle+\text { perms }) ; \\
|19\rangle & =\frac{1}{\sqrt{20}}(|111222\rangle+\text { perms }) ; & |20\rangle & =\frac{1}{\sqrt{20}}(|111333\rangle+\text { perms }) ; \\
|21\rangle & =\frac{1}{\sqrt{20}}(|222333\rangle+\text { perms }) ; & |22\rangle & =\frac{1}{\sqrt{60}}(|111223\rangle+\text { perms }) ; \\
|23\rangle & =\frac{1}{\sqrt{60}}(|111332\rangle+\text { perms }) ; & |24\rangle & =\frac{1}{\sqrt{60}}(|222113\rangle+\text { perms }) ; \\
|25\rangle & =\frac{1}{\sqrt{60}}(|222331\rangle+\text { perms }) ; & |26\rangle & \left.=\frac{1}{\sqrt{60}}|333112\rangle+\text { perms }\right) ; \\
|27\rangle & =\frac{1}{\sqrt{60}}(|333221\rangle+\text { perms }) ; & |28\rangle & =\frac{1}{\sqrt{90}}(|112233\rangle+\text { perms }) .
\end{aligned}
$$

The necessary invariant polynomial is $x^{5} y+y^{5} z+z^{5} x-5 x^{2} y^{2} z^{2}$ [20], which gives a VEV with real components proportional to

$$
v=\left[0,0,0,1,0,0,1,1,0,0,0,0,0,0,0,0,0,0,0,0,0,0,0,0,0,0,0,-\sqrt{\frac{5}{3}} ; 0,0, \ldots 0\right]
$$

i.e., where only $|4\rangle=|7\rangle=|8\rangle$, and $|28\rangle=-\sqrt{\frac{5}{3}} \cdot|4\rangle$ are nonzero.

\footnotetext{
${ }^{5}$ Luhn [55] has shown that the VEV in eq. (3.23) has a $Z_{28}$ symmetry and the vacuum of the potential $V_{15^{\prime}}$ in eq. (2.14) is also symmetric under this symmetry. However, other terms in the Lagrangian will violate this $Z_{28}$, e.g., the Yukawa terms. As it is a discrete symmetry, its breaking can not lead to a pseudo Goldstone boson, but there could be other phenomenological consequences of this $Z_{28}$ that would be interesting to explore.
} 


\begin{tabular}{|c|c|}
\hline Value & Multiplicity \\
\hline 0 & 3 \\
$4 m^{2}$ & 1 \\
$\frac{8 m^{2} \kappa}{5(3 \lambda+\kappa)}$ & 3 \\
\hline
\end{tabular}

Table 1. Scalar mass eigenstates for the SSB pattern $\mathrm{SO}(3) \rightarrow A_{4}$ using a real $\mathbf{7}$ of $\mathrm{SO}(3)$.

\section{Vacuum expectation values and mass spectra}

Thus far, we have discussed how to set up potentials corresponding to specific gauge group representations and then we found vacuum alignments that can potentially be used to break the gauge symmetry to desired discrete subgroups. In this section we minimize the scalar potentials and show where symmetry breaking in the desired directions are allowed. We will find the scale of the symmetry breaking and resulting tree level scalar mass states in terms of the coupling constants of the potential. As usual, the minimization conditions of the potential will lead to constraints on the values of these constants.

\section{1 $\mathrm{SO}(3)$ cases}

\subsection{1 $A_{4}$ scalar spectrum}

We found earlier that a VEV in the direction (3.5) will break $\mathrm{SO}(3)$ to $A_{4}$. The actual VEV is proportional to this direction vector, with the constant of proportionality being the scale of the symmetry breaking. To determine this scale one must minimize the potential (2.2). To achieve this we compute the first derivative with respect to each basis state, insert the alignment from (3.5), and set this equal to zero. This alignment (and all of our alignments below) will give an equation in terms of one basis state (or one linear combination of basis states). For the present case we solve for $|7\rangle$ and take the positive solution to obtain the VEV

$$
\mathcal{V}=\sqrt{\frac{3 m^{2}}{2(3 \lambda+\kappa)}}[0,0,0,0,0,0,1] .
$$

As for any non-trivial stable vacuum, $m^{2}$ must be positive. So to have a real value for our breaking scale, $3 \lambda+\kappa$ must also be positive. We find the scalar mass states by calculating the matrix of second derivatives (the Hessian), inserting the VEV from above, and computing the eigenvalues of the matrix. The resulting values and their multiplicities are given in table 1 .

Looking at table 17 in the appendix, we see that the multiplicities of the eigenvalues match up with the branching of the $\mathbf{7}$ for $\mathrm{SO}(3) \rightarrow A_{4}$, as expected. We see that there are three zero eigenvalues corresponding to the three Goldstone bosons from the breaking of all the generators of $\mathrm{SO}(3)$. Constraints on the coupling constants arise from the requirement that at a minimum of the potential, the eigenvalues must all be positive or zero. Since $m^{2}$ and $3 \lambda+\kappa$ must be positive, requiring the third eigenvalue to be positive leads to the constraint $\kappa>0$ in this case. 


\begin{tabular}{|c|c|}
\hline Value & Multiplicity \\
\hline 0 & 3 \\
$4 m^{2}$ & 1 \\
$\frac{5 m^{2}(5 \kappa+8 \rho-2 \tau)}{7(90 \lambda+10 \kappa+7 \rho+2 \tau)}$ & 3 \\
$\frac{20 m^{2}(5 \kappa+8 \rho-2 \tau)}{7(90 \lambda+10 \kappa+7 \rho+2 \tau)}$ & 2 \\
\hline
\end{tabular}

Table 2. Scalar mass eigenstates for the SSB pattern $\mathrm{SO}(3) \rightarrow S_{4}$ using a real 9 of $\mathrm{SO}(3)$.

\subsection{2 $S_{4}$ scalar spectrum}

For $S_{4}$ we minimize the potential from (2.5) using the alignment (3.7). We obtain a VEV

$$
\mathcal{V}=\sqrt{\frac{25 m^{2}}{4(90 \lambda+10 \kappa+7 \rho+2 \tau)}}\left[\sqrt{\frac{7}{5}}, 0,0,0,0,0,0,1,0\right] .
$$

Hence a real value for the symmetry breaking scale requires $90 \lambda+10 \kappa+7 \rho+2 \tau>0$. The scalar mass states ${ }^{6}$ are found in table 2 and are all non-negative if

$$
5 \kappa+8 \rho-2 \tau>0
$$

is also satisfied. The three zeros correspond to the broken $\mathrm{SO}(3)$ generators.

\subsection{3 $\quad A_{5}$ scalar spectrum}

For $A_{5}$, we minimize the potential from (2.6) using the alignment (3.9). We obtain a VEV

$$
\begin{aligned}
\mathcal{V}=\sqrt{\frac{1155 m^{2}}{128(\lambda+140 \kappa+84 \rho+65 \tau+14 \nu+9 \sigma-2 \chi)}} \\
\times\left[1,0,0,-\sqrt{\frac{21}{2}}, 0,0,0,0,0,0, \sqrt{\frac{105}{22}}, 0\right] .
\end{aligned}
$$

A real value for our breaking scale requires $420 \lambda+140 \kappa+84 \rho+65 \tau+14 \nu+9 \sigma-2 \chi>0$. The scalar mass states ${ }^{7}$ are given in table 3 .

Again we have the three zero eigenvalues corresponding to the broken $\mathrm{SO}(3)$ generators. To have non-negative masses we must satisfy the constraints

$$
105 \kappa+196 \rho+240 \tau-14 \nu-19 \sigma+12 \chi>0,
$$

and

$$
14 \rho+45 \tau+14 \nu-11 \sigma+18 \chi>0 .
$$

\footnotetext{
${ }^{6}$ In order to normalize the eigenvalues for $S_{4}$ to those in other cases when we do not use the spherical harmonic basis, we have multiplied all quadratic terms by a factor of $\frac{1}{8}$ and quartic terms by a factor of $\frac{1}{64}$.

${ }^{7}$ Again by expressing the states in terms of spherical harmonics, we obtain different normalizations for our basis states which lead to a different normalization scale for the VEV scale and scalar mass states. To correct this for $A_{5}$ we have multiplied the quadratic term by a factor of $\frac{5}{352}$ and the quartic terms by $\left(\frac{5}{352}\right)^{2}$ so that our states are now normalized the same way as our other breakings.
} 


\begin{tabular}{|c|c|}
\hline Value & Multiplicity \\
\hline 0 & 3 \\
$4 m^{2}$ & 1 \\
$\frac{28 m^{2}(105 \kappa+196 \rho+240 \tau-14 \nu-19 \sigma+12 \chi)}{33(420 \lambda+140 \kappa+84 \rho+65 \tau+14 \nu+9 \sigma-2 \chi}$ & 5 \\
$\frac{28 m^{2}(14 \rho+45 \tau+14 \nu-11 \sigma+18 \chi)}{33(420 \lambda+140 \kappa+84 \rho+65 \tau+14 \nu+9 \sigma-2 \chi}$ & 4 \\
\hline
\end{tabular}

Table 3. Scalar mass eigenstates for the SSB pattern $\mathrm{SO}(3) \rightarrow A_{5}$ using a real $\mathbf{1 3}$ of $\mathrm{SO}(3)$.

\begin{tabular}{|c|c|}
\hline Value & Multiplicity \\
\hline 0 & 4 \\
$4 m^{2}$ & 1 \\
$\frac{2 m^{2} \kappa}{3(2 \lambda+\kappa+\rho+\tau)}$ & 2 \\
$\frac{4 m^{2}(\kappa+\rho+\tau)}{2 \lambda+\kappa+\rho+\tau}$ & 1 \\
$\frac{-3 m^{2}(2 \rho+3 \tau)}{5(2 \lambda+\kappa+\rho+\tau)}$ & 1 \\
$\frac{-2 m^{2}(2 \rho+3 \tau)}{5(2 \lambda+\kappa+\rho+\tau)}$ & 2 \\
$\frac{-m^{2}(6 \rho+7 \tau)}{5(2 \lambda+\kappa+\rho+\tau)}$ & 1 \\
$\frac{-2 m^{2}(8 \rho+9 \tau)}{15(2 \lambda+\kappa+\rho+\tau)}$ & 2 \\
\hline
\end{tabular}

Table 4. Scalar mass eigenstates for the SSB pattern SU(2) $\rightarrow Q_{6}$ using a complex $\mathbf{7}$ of SU(2).

\section{$4.2 \mathrm{SU}(2)$ cases}

\subsection{1 $Q_{6}$ scalar spectrum}

We break the symmetry of the potential given in eq. (2.7) with the alignment in eq. (3.12) to obtain a VEV

$$
\mathcal{V}=\sqrt{\frac{m^{2}}{2(2 \lambda+\kappa+\rho+\tau)}}[1,1,0,0,0,0,0 ; 0,0,0,0,0,0,0] .
$$

Thus we require $\kappa+2 \lambda+\rho+\tau>0$. The eigenvalues of the Hessian are given in table 4 . The constraints from these mass eigenvalues are

$$
\begin{aligned}
& \kappa>0, \\
& \kappa>-(\rho+\tau),
\end{aligned}
$$

and

$$
2 \rho+3 \tau, 6 \rho+7 \tau, 8 \rho+9 \tau<0 .
$$

There are clearly stable minima when $\lambda>0, \kappa>0, \rho<0$ and $\tau<0$. The extra zero eigenvalue comes from breaking an accidental $\mathrm{U}(1)$ phase symmetry. This gives rise to a pseudo-goldstone boson that can gain a mass through quantum corrections. 


\begin{tabular}{|c|c|}
\hline Value & Multiplicity \\
\hline 0 & 4 \\
$4 m^{2}$ & 1 \\
$\frac{12 m^{2} \tau}{5(12 \lambda+6 \kappa+4 \rho+3 \tau)}$ & 3 \\
$\frac{16 m^{2}(2 \rho+3 \tau)}{5(12 \lambda+6 \kappa+4 \rho+3 \tau)}$ & 3 \\
$\frac{4 m^{2}(8 \kappa+8 \rho+9 \tau)}{3(12 \lambda+6 \kappa+4 \rho+3 \tau)}$ & 3 \\
\hline
\end{tabular}

Table 5. Scalar mass eigenstates for the SSB pattern SU(2) $\rightarrow T^{\prime}$ using a complex $\mathbf{7}$ of SU(2).

\subsection{2 $\quad T^{\prime}$ scalar spectrum}

The potential and the vacuum alignment of the breaking of $\mathrm{SU}(2)$ to $\mathrm{T}^{\prime}$ with a real $\mathbf{7}$ are the same as for $\mathrm{SO}(3) \rightarrow A_{4}$. Therefore the breaking scale and the mass states will be exactly the same, as the two models can only be differentiated by the non-scalar part of the Lagrangian.

For a the breaking with a complex 7 we minimize the potential in eq. (2.7) but this time using the alignment eq. (3.11) to obtain the VEV

$$
\mathcal{V}=\sqrt{\frac{3 m^{2}}{(12 \lambda+6 \kappa+4 \rho+3 \tau)}}[0,-1,0,0,0,1,0 ; 0,0,0,0,0,0,0]
$$

which leads to the constraint that $12 \lambda+6 \kappa+4 \rho+3 \tau>0$. The eigenvalues of the Hessian are shown in table 5 .

From the requirement of positive eigenvalues we deduce the constraints

$$
\begin{aligned}
& \tau>0, \\
& \rho>-\frac{3}{2} \tau,
\end{aligned}
$$

and

$$
\frac{3}{8} \tau>\kappa>-8 \rho-\frac{9}{8} \tau
$$

As in the $Q_{6}$ example, the extra zero eigenvalue is a result of breaking the $\mathrm{U}(1)$ phase symmetry in the potential.

\subsection{3 $O^{\prime}$ scalar spectrum}

The breaking scale and scalar mass spectrum of $\mathrm{SU}(2)$ to $O^{\prime}$ with a real $\mathbf{9}$ is exactly the same as that for $\mathrm{SO}(3)$ to $S_{4}$, where differences between two models would come from the non-scalar part of the Lagrangian. 


\begin{tabular}{|c|c|}
\hline Value & Multiplicity \\
\hline 0 & 4 \\
$4 m^{2}$ & 1 \\
$\frac{-24 m^{2} \sigma}{7(60 \lambda+30 \kappa+20 \rho+15 \tau+14 \sigma)}$ & 2 \\
$\frac{5 m^{2}(10 \rho+15 \tau+16 \sigma)}{7(60 \lambda+30 \kappa+20 \rho+15 \tau+14 \sigma)}$ & 3 \\
$\frac{20 m^{2}(10 \rho+15 \tau+16 \sigma)}{7(60 \lambda+30 \kappa+20 \rho+15 \tau+14 \sigma)}$ & 2 \\
$\frac{2 m^{2}(25 \kappa+25 \rho+25 \tau+24 \sigma)}{60 \lambda+30 \kappa+20 \rho+15 \tau+14 \sigma}$ & 3 \\
$\frac{3 m^{2}(25 \tau+32 \sigma)}{7(60 \lambda+30 \kappa+20 \rho+15 \tau+14 \sigma)}$ & 3 \\
\hline
\end{tabular}

Table 6. Scalar mass eigenstates for the SSB pattern $\mathrm{SU}(2) \rightarrow O^{\prime}$ using a complex 9 of $\mathrm{SU}(2)$.

For a complex 9 we minimize the potential in eq. (2.8) using the alignment eq. (3.14) and obtain a VEV

$$
\begin{aligned}
\mathcal{V}=\sqrt{\frac{25 m^{2}}{4(60 \lambda+30 \kappa+20 \rho+15 \tau+14 \sigma)}} \\
\times\left[1,1,0,0,0,0,0,0, \frac{14}{\sqrt{70}} ; 0,0,0,0,0,0,0,0,0\right] .
\end{aligned}
$$

Thus $60 \lambda+30 \kappa+20 \rho+15 \tau+14 \sigma$ must be $>0$. Looking at table 6 , we see that there are 3 zeros corresponding to the 3 broken $\mathrm{SU}(2)$ generators, as well as an extra zero from breaking the $\mathrm{U}(1)$ phase symmetry.

We have the additional constraints

$$
\begin{aligned}
\sigma & <0, \\
10 \rho+15 \tau+16 \sigma & >0, \\
25 \kappa+25 \rho+25 \tau+24 \sigma & >0,
\end{aligned}
$$

and

$$
25 \tau+32 \sigma>0
$$

\subsection{4 $\quad I^{\prime}$ scalar spectrum}

Simlar to the cases of $T^{\prime}$ and $O^{\prime}$, the breaking scale and mass spectrum of a real 13 of $\mathrm{SU}(2)$ to $I^{\prime}$ are the same of that of $\mathrm{SO}(3)$ to $A_{5}$ with a real 13.

For a complex 13 we minimize the potential of eq. (2.9) using the alignment eq. (3.16) and obtain a VEV

$$
\begin{aligned}
\mathcal{V}=7 \sqrt{\frac{6 m^{2}}{5(420 \lambda+210 \kappa+140 \rho+105 \tau+84 \nu+70 \sigma+65 \chi)}} \\
\quad \times\left[0,0,1,-1,0,0,0,0,0,0,0,0, \sqrt{\frac{11}{7}} ; 0,0,0,0,0,0,0,0,0,0,0,0,0\right] .
\end{aligned}
$$




\begin{tabular}{|c|c|}
\hline Value & Multiplicity \\
\hline 0 & 4 \\
$4 m^{2}$ & 1 \\
$\frac{28 m^{2}(14 \nu+35 \sigma+45 \chi)}{33(420 \lambda+210 \kappa+140 \rho+105 \tau+84 \nu+70 \sigma+65 \chi)}$ & 4 \\
$\frac{5 m^{2}(49 \sigma+72 \chi)}{33(420 \lambda+210 \kappa+140 \rho+105 \tau+84 \nu+70 \sigma+65 \chi)}$ & 5 \\
$\frac{14 m^{2}(210 \rho+315 \tau+392 \nu+455 \sigma+480 \chi)}{33(420 \lambda+210 \kappa+140 \rho+105 \tau+84 \nu+70 \sigma+65 \chi)}$ & 5 \\
$\frac{m^{2}(980 \kappa+980 \rho+882 \tau+784 \nu+735 \sigma+720 \chi)}{3(420 \lambda+210 \kappa+140 \rho+105 \tau+84 \nu+70 \sigma+65 \chi)}$ & 3 \\
$\frac{4 m^{2}(441 \tau+882 \nu+1225 \sigma+1350 \chi)}{33(420 \lambda+210 \kappa+140 \rho+105 \tau+84 \nu+70 \sigma+65 \chi)}$ & 4 \\
\hline
\end{tabular}

Table 7. Scalar mass eigenstates for the SSB pattern $\mathrm{SU}(2) \rightarrow I^{\prime}$ using a complex $\mathbf{1 3}$ of $\mathrm{SU}(2)$.

Thus $420 \lambda+210 \kappa+140 \rho+105 \tau+84 \nu+70 \sigma+65 \chi$ must be positive. The eigenvalues of the Hessian (see table 7 ) are all real and positive semidefinite for positive scalar quartic couplings. (More detailed constraints on the scalar quartics can clearly be extracted from the individual mass eigenvalues.) There are 3 zeros corresponding to the 3 broken SU(2) generators, as well as an extra zero from breaking the $\mathrm{U}(1)$ phase symmetry.

\section{3 $\mathrm{SU}(3)$ cases}

\subsection{1 $A_{4}$ scalar spectrum}

For the nonminimal breaking $\mathrm{SU}(3) \rightarrow A_{4}$ we minimize the potential eq. (2.11) and use the alignment eq. (3.18) to get the VEV [19]

$$
\begin{aligned}
& \mathcal{V}=\sqrt{\frac{m^{2}}{2(3 \lambda+\eta+\kappa+\rho+\tau)}} \\
& \quad \times[0,0,0,0,0,0,0,0,0,0,0,0,1,1,1 ; 0,0,0,0,0,0,0,0,0,0,0,0,0,0,0]
\end{aligned}
$$

Thus $3 \lambda+\eta+\kappa+\rho+\tau$ must be positive. The eigenvalues of the Hessian are shown in table 8 .

We expect eight zeros corresponding to the broken generators of $\mathrm{SU}(3)$, but again an extra zero eigenvalue arises from breaking the accidental $\mathrm{U}(1)$ phase symmetry. As for constraints, we can readily see that

$$
\begin{gathered}
\eta<0 \\
5 \kappa+2 \rho+4 \tau>\sqrt{(4 \tau+2 \rho-3 \kappa)^{2}+16(\rho+\kappa+2 \eta)^{2}},
\end{gathered}
$$

and

$$
3 \kappa-5 \eta-2 \rho+4 \tau>\frac{1}{3} \sqrt{(9 \eta-7 \kappa+10 \rho-4 \tau)^{2}+8(\rho+2 \kappa-4 \tau)^{2}}
$$

are required. An example of where all these constraints can be satisfied is

$$
2 \rho=3 \kappa, \rho+\kappa=-2|\eta|, \text { and } 5 \kappa+3 \rho>0, \text { where } \kappa, \rho, \text { and } \tau>0 .
$$




\begin{tabular}{|c|c|}
\hline Value & Multiplicity \\
\hline 0 & 9 \\
$4 m^{2}$ & 1 \\
$\frac{m^{2}(-2 \eta+\kappa-2 \rho+4 \tau)}{3 \lambda+\eta+\kappa+\rho+\tau}$ & 2 \\
$\frac{-3 m^{2} \eta}{3 \lambda+\eta+\kappa+\rho+\tau}$ & 6 \\
$\frac{m^{2}}{4(3 \lambda+\eta+\kappa+\rho+\tau)}\left(5 \kappa+2 \rho+4 \tau+\sqrt{(4 \tau+2 \rho-3 \kappa)^{2}+16(\rho+\kappa+2 \eta)^{2}}\right)$ & 3 \\
$\frac{m^{2}}{4(3 \lambda+\eta+\kappa+\rho+\tau)}\left(5 \kappa+2 \rho+4 \tau-\sqrt{(4 \tau+2 \rho-3 \kappa)^{2}+16(\rho+\kappa+2 \eta)^{2}}\right)$ & 3 \\
$\frac{m^{2}}{2(3 \lambda+\eta+\kappa+\rho+\tau)}\left(3 \kappa-5 \eta-2 \rho+4 \tau+\frac{1}{3} \sqrt{(9 \eta-7 \kappa+10 \rho-4 \tau)^{2}+8(\rho+2 \kappa-4 \tau)^{2}}\right)$ & 3 \\
$\frac{m^{2}}{2(3 \lambda+\eta+\kappa+\rho+\tau)}\left(3 \kappa-5 \eta-2 \rho+4 \tau-\frac{1}{3} \sqrt{(9 \eta-7 \kappa+10 \rho-4 \tau)^{2}+8(\rho+2 \kappa-4 \tau)^{2}}\right)$ & 3 \\
\hline
\end{tabular}

Table 8. Scalar mass eigenstates for the SSB pattern $\mathrm{SU}(3) \rightarrow A_{4}$ using a $\mathbf{1 5}$ of $\mathrm{SU}(3)$.

\begin{tabular}{|c|c|}
\hline Value & Multiplicity \\
\hline 0 & 9 \\
$4 m^{2}$ & 1 \\
$\frac{2(2 \kappa-\rho+2 \tau) m^{2}}{\kappa+3 \lambda+\rho+\tau}$ & 2 \\
$\frac{m^{2}}{12(3 \lambda+\kappa+\rho+\tau)} \times \alpha$ \\
$\frac{m^{2}}{12(3 \lambda+\kappa+\rho+\tau)} \times \beta$ \\
$\frac{m^{2}}{12(3 \lambda+\kappa+\rho+\tau)} \times \gamma$ & 6 \\
\hline
\end{tabular}

Table 9. Scalar mass eigenstates for the SSB pattern $\mathrm{SU}(3) \rightarrow T_{7}$ using a $\mathbf{1 5}$ of $\mathrm{SU}(3)$.

\subsection{2 $\quad T_{7}$ scalar spectrum}

For this breaking we again minimize eq. (2.11), now using the alignment eq. (3.19) to obtain the VEV [19]

$$
\begin{aligned}
& \mathcal{V}=\sqrt{\frac{m^{2}}{2(3 \lambda+\kappa+\rho+\tau)}} \\
& \quad \times[0,0,0,0,0,0,0,0,0,0,0,0,1,1,1 ; 0,0,0,0,0,0,0,0,0,0,0,0,0,0,0] .
\end{aligned}
$$

Thus $3 \lambda+\kappa+\rho+\tau$ must be positive. The eigenvalues of the Hessian are shown in table 9 , where $\alpha, \beta, \gamma$ are the three roots of the polynomial $10368 \eta^{2}(\rho-\kappa-\tau)+3888 \eta \rho^{2}-$ $15552 \eta \kappa \tau+\left(648 \eta^{2}+972 \eta \kappa-648 \eta \rho-180 \rho^{2}+1296 \eta \tau+720 \kappa \tau\right) x+(6 \rho-54 \eta-21 \kappa-36 \tau) x^{2}+x^{3}$. We have the constraints

$$
2 \kappa-\rho+2 \tau>0
$$

and

$$
\alpha, \beta, \gamma>0
$$




\begin{tabular}{|c|c|}
\hline Value & Multiplicity \\
\hline 0 & 11 \\
$4 m^{2}$ & 1 \\
$\frac{4 \kappa m^{2}}{3 \lambda+\kappa}$ & 2 \\
$\frac{\kappa m^{2}}{3(3 \lambda+\kappa)}$ & 6 \\
\hline
\end{tabular}

Table 10. Scalar mass eigenstates for the SSB pattern $\mathrm{SU}(3) \rightarrow \Delta(27)$ using a $\mathbf{1 0}$ of $\mathrm{SU}(3)$.

The extra zero is once again due to breaking the $\mathrm{U}(1)$ phase symmetry. We cannot remedy this by including cubic terms this time, because we need the couplings on those terms to vanish in order to have a stable minimum. Numerical studies show that there is a range of scalar quartic coupling constant values where the minimum is stable. An example of such numerical analysis will be discussed below.

\subsection{3 $\Delta(27)$ scalar spectrum}

Minimizing the potential of eq. (2.12) with the alignment eq. (3.21) we obtain a VEV

$$
\mathcal{V}=\sqrt{\frac{m^{2}}{2(3 \lambda+\kappa)}}[1,1,1,0,0,0,0,0,0,0 ; 0,0,0,0,0,0,0,0,0,0]
$$

giving the constraint $3 \lambda+\kappa>0$. The eigenvalues of the Hessian are in table 10, from which we see that $\kappa>0$ is required. Again we have an extra zero from an accidental U(1), and in this case the cubic terms vanish upon summation. The two extra zeros are the result of an additional $\Delta(27)$ singlet within the $\mathbf{1 0}$. (For a detailled explanation see [19].)

\subsubsection{PSL $(2,7)$ scalar spectrum}

Minimizing the potential of eq. (2.14) with the alignment eq. (3.23) we obtain a VEV

$$
\mathcal{V}=\sqrt{\frac{m^{2}}{6 \lambda+2 \kappa+\rho}}[0,0,0,0,1,1,0,0,1,0,0,0,0,0,0 ; 0,0,0,0,0,0,0,0,0,0,0,0,0,0,0]
$$

Thus $6 \lambda+2 \kappa+\rho>0$. The eigenvalues of the Hessian are found in table 11 from which we get the constraints

$$
\rho>0, \quad \text { and } \quad 7 \kappa+8 \rho>0 .
$$

We once again have an extra zero, but this time it is possible to include cubic terms to break the $\mathrm{U}(1)$ phase. The two cubic terms we can include are

$$
\begin{gathered}
\epsilon_{i m q} \epsilon_{j n r} \epsilon_{k o s} \epsilon_{l p t} T^{i j k l} T^{m n o p} T^{q r s t}, \\
\text { and } \\
\epsilon^{i m q} \epsilon^{j n r} \epsilon^{k o s} \epsilon^{l p t} T_{i j k l} T_{m n o p} T_{q r s t},
\end{gathered}
$$




\begin{tabular}{|c|c|}
\hline Value & Multiplicity \\
\hline 0 & 9 \\
$4 m^{2}$ & 1 \\
$\frac{(7 \kappa+8 \rho) m^{2}}{2(6 \lambda+2 \kappa+\rho)}$ & 8 \\
$\frac{2(3-\sqrt{2}) \rho m^{2}}{3(6 \lambda+2 \kappa+\rho)}$ & 6 \\
$\frac{2(3+\sqrt{2}) \rho m^{2}}{3(6 \lambda+2 \kappa+\rho)}$ & 6 \\
\hline
\end{tabular}

Table 11. Scalar mass eigenstates for the SSB pattern $\mathrm{SU}(3) \rightarrow \operatorname{PSL}(2,7)$ using a $\mathbf{1 5}^{\prime}$ of $\mathrm{SU}(3)$.

\begin{tabular}{|c|c|}
\hline Value & Multiplicity \\
\hline 0 & 8 \\
4.002 & 1 \\
.006003 & 1 \\
0.323647 & 6 \\
0.125244 & 6 \\
0.838169 & 8 \\
\hline
\end{tabular}

Table 12. Numerical results where cubic terms are included for the scalar mass eigenstates of the $\mathrm{SSB}$ pattern $\mathrm{SU}(3) \rightarrow \mathrm{PSL}(2,7)$ using a $\mathbf{1 5}^{\prime}$ of $\mathrm{SU}(3)$.

which are Hermitian conjugates and are included in the potential with the same real coupling constant, $\zeta$. The VEV scale for the potential including the cubic is now

$$
\frac{-3 \zeta \pm \sqrt{9 \zeta^{2}+4 m^{2}(6 \lambda+2 \kappa+\rho)}}{2(6 \lambda+2 \kappa+\rho)} .
$$

Notice that there may be two possible solutions. The constraint that must hold in both cases is $9 \zeta^{2}+4 m^{2}(2 \kappa+6 \lambda+\rho) \geq 0$.

Calculating the eigenvalues of the Hessian produces solutions involving the roots of a polynomial which is much too large to display, but it is notable that it does produce 8 zeros rather than 9. Furthermore, following the usual procedure, but this time numerically where for simplicity setting all quartic coupling constants to unity, the quadratic coupling to -1 and the cubic to .001 (these values are selected to ensure a stable minimum) produces a VEV scale of approximately 0.3335 and eigenvalues whose multiplicities match the branching rules $\mathrm{SU}(3) \rightarrow \operatorname{PSL}(2,7)$, as shown in table 12 . The degeneracy of the pseudo-Goldstone mass with that of the true Goldstones is lifted as expected, as can be seen in table 12. Finally note that although we have set all the coupling constants except for the cubic to integer values, we can easily rescale them to smaller values to be sure we are in the perturbative regime of the theory without disturbing the stability of the result. Specifically, while the scalar quartic couplings in the numerical example are not in 


\begin{tabular}{|c|c|}
\hline Value & Multiplicity \\
\hline 0 & 16 \\
$4 m^{2}$ & 1 \\
$\frac{4(7 \rho+9 \tau) m^{2}}{5(42 \lambda+14 \kappa+7 \rho+6 \tau)}$ & 7 \\
$\frac{(21 \kappa+20 \rho+18 \tau) m^{2}}{42 \lambda+14 \kappa+7 \rho+6 \tau}$ & 8 \\
$\frac{1}{200(42 \lambda+14 \kappa+7 \rho+6 \tau)^{2}} \times A$ & 6 \\
$\frac{1}{200(42 \lambda+14 \kappa+7 \rho+6 \tau)^{2}} \times B$ & 6 \\
$\frac{1}{200(42 \lambda+14 \kappa+7 \rho+6 \tau)^{2}} \times C$ & 6 \\
$\frac{1}{200(42 \lambda+14 \kappa+7 \rho+6 \tau)^{2}} \times D$ & 6 \\
\hline
\end{tabular}

Table 13. Scalar mass eigenstates for the SSB pattern $\mathrm{SU}(3) \rightarrow \operatorname{PSL}(2,7)$ using a $\mathbf{2 8}$ of $\mathrm{SU}(3)$.

the perturbative range, we can rescale all the quartics by a factor $s$ and $\zeta$ by a factor $\sqrt{s}$. This leaves the eigenvalues unchanged and puts us into the perturbative regime.

Moving on to the $\mathbf{2 8}$, we minimize the potential in eq. (2.15) with the alignment eq. (3.25) to obtain a VEV

$$
\mathcal{V}=\sqrt{\frac{9 m^{2}}{2(42 \lambda+14 \kappa+7 \rho+6 \tau)}}\left[0,0,0,1,0,0,1,1,0,0, \ldots 0,0,-\sqrt{\frac{5}{3}} ; 0,0, \ldots 0\right] .
$$

Thus $42 \lambda+14 \kappa+7 \rho+6 \tau>0$. The eigenvalues of the Hessian are given in table 13 Further constraints are

$$
\begin{aligned}
7 \rho+9 \tau & >0, \\
21 \kappa+20 \rho+18 \tau & >0, \\
\text { and } \quad A, B, C, D & >0 .
\end{aligned}
$$

where A, B, C, and D are the roots of a very large quartic polynomial. Numerical work shows that all four roots can be positive, simultaneously leading to all positive eigenvalues in table 13 and a stable minimum when the other constraints are also satisfied. We see that there are eight zeros from the broken generators of $\mathrm{SU}(3)$, and one zero from breaking the broken U(1) phase. But unique to this breaking we have seven extra zeros, which implies that there are seven more broken generators from an accidental symmetry of the Lagrangian that we have so far been unable to identify, leading to a total of 8 pseudo-Goldstone bosons.

\subsection{Symmetry breaking summary}

Let us briefly summarize our results. We have shown that we can break from $G$ to $\Gamma$ for the gauge and discrete groups listed in the introduction. The minima can be stable since none of the eigenvalues of the scalars are negative for allowed regions of parameter space. Zero eigenvalues correspond to Goldstone bosons in each case and to additional pseudoGoldstone bosons in several cases. Specifically for the cases we have studied of $\mathrm{SO}(3)$ 


\begin{tabular}{|c|c|}
\hline SSB pattern & decomposition \\
\hline $\mathrm{SO}(3) \rightarrow A_{4}$ & $\mathbf{7} \rightarrow \mathbf{1}+\mathbf{3}_{\mathrm{G}}+\mathbf{3}$ \\
$\mathrm{SO}(3) \rightarrow S_{4}$ & $\mathbf{9} \rightarrow \mathbf{1}+\mathbf{2}+\mathbf{3}_{\mathrm{G}}+\mathbf{3}$ \\
$\mathrm{SO}(3) \rightarrow A_{5}$ & $\mathbf{1 3} \rightarrow \mathbf{1}+\mathbf{3}_{\mathrm{G}}+\mathbf{4}+\mathbf{5}$ \\
\hline
\end{tabular}

Table 14. Scalar mass eigenstates for the $\mathrm{SSB}$ patterns $\mathrm{SO}(3) \rightarrow A_{4}, S_{4}$ and $A_{5}$ using real symmetric tensor irreps of $\mathrm{SO}(3)$.

\begin{tabular}{|c|c|}
\hline $\mathrm{SSB}$ pattern & decomposition \\
\hline $\mathrm{SU}(2) \rightarrow Q_{6}$ & $\mathbf{7}_{\mathbf{c}} \rightarrow \mathbf{1}+\mathbf{1}^{\prime}+\left(\mathbf{1}^{\prime}+\mathbf{2}^{\prime}\right)_{\mathrm{G}}+\mathbf{2}^{\prime}+\mathbf{1}_{\mathrm{pGB}}+\mathbf{1}^{\prime}+\mathbf{1}^{\prime}+\mathbf{2}^{\prime}+\mathbf{2}^{\prime}$ \\
$\mathrm{SU}(2) \rightarrow T^{\prime}$ & $\mathbf{7}_{\mathbf{c}} \rightarrow \mathbf{1}+\mathbf{3}_{\mathrm{G}}+\mathbf{3}+\mathbf{1}_{\mathrm{pGB}}+\mathbf{3}+\mathbf{3}$ \\
$\mathrm{SU}(2) \rightarrow O^{\prime}$ & $\mathbf{9}_{\mathbf{c}} \rightarrow \mathbf{1}+\mathbf{2}+\mathbf{3}_{\mathrm{G}}+\mathbf{3}+\mathbf{1}_{\mathrm{pGB}}+\mathbf{2}+\mathbf{3}+\mathbf{3}$ \\
$\mathrm{SU}(2) \rightarrow I^{\prime}$ & $\mathbf{1 3}_{\mathbf{c}} \rightarrow \mathbf{1}+\mathbf{3}_{\mathrm{G}}+\mathbf{4}+\mathbf{5}+\mathbf{1}_{\mathrm{pGB}}+\mathbf{3}+\mathbf{4}+\mathbf{5}$ \\
\hline
\end{tabular}

Table 15. Scalar mass eigenstates for the SSB patterns $\mathrm{SU}(2) \rightarrow Q_{6}, T^{\prime}, O^{\prime}$ and $I^{\prime}$ using symmetric tensor irreps of $\mathrm{SU}(2)$, i.e., complexified symmetric tensor irreps of $\mathrm{SO}(3)$.

breaking to a discrete symmetry the results are summarized in table 14 . The $G$ subscript indicates the Goldstones. In each case the masses of the particles in different discrete group irreps are all different, so the initial degeneracy of the scalar masses is lifted to the extent allowed by the discrete group. For the cases of SU(2) breaking to discrete symmetries, the results are summarized in table 15. Again all the discrete group irreps correspond to different masses except for the zero eigenvalue states where we have indicated the true Goldstones and the pseudo-Goldstones (by subscripts pGB) due to breaking of the phase symmetry on the potentials. The subscript $c$ indicates that the irreps are complexified and the decompositions are written in terms of real components. The results begin to become more complicated for the $\mathrm{SU}(3)$ cases we have investigated, and this can be seen in table 16. Now some irreps masses have become degenerate and we have indicated these cases by collecting those discrete group irreps with parentheses and labeling the collection with a deg. subscript. All the cases have a pseudo-Goldstones associated with breaking of phase invariance. The breaking to $T_{7}$ with a $\mathbf{1 0}$ leads to two additional pseudo-Goldstones as discussed in [19] and the breaking to $\operatorname{PSL}(2,7)$ with a $\mathbf{2 8}$ has seven additional pseudoGoldstones. Since the $\mathbf{2 8}$ was derived from $\mathbf{3}^{\mathbf{6}}$ one could conjecture that the potential has a $\operatorname{Spin}(6) \sim \mathrm{SU}(4)$ accidental symmetry that contains the gauged SU(3), and that the VEV breaks all $15 \mathrm{SU}(4)$ plus the phase to give a total of 16 massless states. Finally, recall that for the breaking to $\operatorname{PSL}(2,7)$ with a $\mathbf{1 5}^{\prime}$ we have shown that phase symmetry can be avoided if we add cubic terms, hence there is no pseudo-Goldstone after SSB in that case, see table 12 .

\section{Discussion and conclusion}

The standard model includes 28 unspecified parameters, some of which describe fermion masses and mixing angles. Consequently, we do not know why the quark and lepton masses and mixings are what they are. To fix these parameters, a standard approach has been to extend the SM by a discrete symmetry, but this approach is not without its difficulties 


\begin{tabular}{|c|c|}
\hline SSB pattern & decomposition \\
\hline $\mathrm{SU}(3) \rightarrow A_{4}$ & $15 \rightarrow 1+\left(1^{\prime}+1^{\prime \prime}+3+3\right)_{\mathrm{G}}+(3+3)_{\mathrm{deg} .}+\mathbf{1}_{\mathrm{pGB}}+\left(\mathbf{1}^{\prime}+\mathbf{1}^{\prime \prime}\right)_{\mathrm{deg} .}+3+3+3+3$ \\
\hline $\mathrm{SU}(3) \rightarrow T_{7}$ & $15 \longrightarrow 1+\left(1^{\prime}+1^{\prime \prime}+3^{\prime}+3^{\prime \prime}\right)_{\mathrm{G}}+\left(3^{\prime}+3^{\prime \prime}\right)_{\mathrm{deg}}$ \\
\hline & $+\mathbf{1}_{\mathrm{pGB}}+\left(\mathbf{1}^{\prime}+\mathbf{1}^{\prime \prime}\right)_{\mathrm{deg} .}+\left(\mathbf{3}^{\prime}+\mathbf{3}^{\prime \prime}\right)_{\mathrm{deg} .}+\left(\mathbf{3}^{\prime}+\mathbf{3}^{\prime \prime}\right)_{\mathrm{deg}}$ \\
\hline $\mathrm{SU}(3) \rightarrow \Delta(27)$ & $10 \rightarrow 1+1_{\mathrm{pGB}}+\left(\Sigma_{\mathrm{n}=2}^{9}\right)_{\mathrm{G}}+3+(1+1)_{\mathrm{pGB}}+\left(\mathbf{1}_{2}+\mathbf{1}_{3}\right)_{\mathrm{deg} .}+\left(\Sigma_{\mathrm{n}=4}^{9}\right)_{\mathrm{deg}}$. \\
\hline $\mathrm{SU}(3) \rightarrow \operatorname{PSL}(2,7)$ & $15^{\prime} \rightarrow 1+6+8_{\mathrm{G}}+1_{\mathrm{pGB}}+6+8$ \\
\hline $\mathrm{SU}(3) \rightarrow \operatorname{PSL}(2,7)$ & $28 \rightarrow 1+6+6+7+8_{\mathrm{G}}+1_{\mathrm{pGB}}+6+6+7_{\mathrm{pGB}}+8$ \\
\hline
\end{tabular}

Table 16. Scalar mass eigenstates for the SSB patterns $\mathrm{SU}(3) \rightarrow A_{4}, T_{7}, \Delta(27)$ and $\mathrm{PSL}(2,7)$ using symmetric tensor irreps of $\mathrm{SU}(3)$, except for the $\mathbf{1 5}$ which is of mixed symmetry.

as discussed in the Introduction. What would seem more natural would be to increase the gauge group to $\mathrm{SU}(3) \times \mathrm{SU}(2) \times \mathrm{U}(1) \times G$ and extend the scalar sector. Then this model can be of the same general type as the SM, i.e., an anomaly-free gauge theory with fermions that gets spontaneously broken by VEVs of scalar fields. If the SSB of $G$ results in a discrete subgroup $\Gamma$ then we arrive at a $\mathrm{SU}(3) \times \mathrm{SU}(2) \times \mathrm{U}(1) \times \Gamma$ via a route that avoids the problems just mentioned, without choosing an ad hoc discrete group for extending the SM.

The region where a certain VEV corresponds to a minimum of a potential depends on the constraints on the values of the coupling constants. If the constraints are violated, then one or more of the scalar mass squared eigenvalues becomes negative indicating an instability. To resolve the instability the VEV must orient itself in a new direction where all the mass eigenvalues are again positive. Since the potentials we consider are in general fairly complicated, involving numerous coupling constants, we would expect to find several minima for each potential. Hence a complete exploration would require studying the coupling constant constraints in the full multidimensional parameter space for each potential. Here we have dealt only with renormalizable dimension four potentials, and their tree level minima. More generally, we could include running of the coupling constant with energy scale or temperature. Such running could cause a phase transition carrying us from one minimum to another. Let us consider just one specific example, the potential $V_{7_{c}}$. Amongst other possibilities, it can be used to break $\mathrm{SU}(2)$ to $Q_{6}$ or to $T^{\prime}$. The $Q_{6}$ breaking requires we satisfy the constraint $2 \rho+3 \tau<0$, while breaking to $T^{\prime}$ requires $2 \rho+3 \tau>0$. (See tables 4 and 5.) There is a domain wall at the boundary where $2 \rho+3 \tau=0$, where both cases have three extra eigenvalues going to zero. Near the boundary loop corrections become important and one can see that if the coupling constant run so that this boundary is crossed, then there is a phase transition from one symmetry to the other. This happens smoothly if the phase transition is second order but the field could get hung up in a local minimum if the phase transition is first order.

Here, based on the techniques of Luhn [19] and Merle and R. Zwicky [20], we have demonstrated that we can carry out the $G \rightarrow \Gamma$ SSB in many cases of interest, specifically breaking to $A_{4}, S_{4}, A_{5}, Q_{6}, T^{\prime}, O^{\prime}, I^{\prime}, T_{7}, \Delta(27)$ and $\operatorname{PSL}(2,7)$. Other cases can be handled by the same techniques. Many other discrete groups have been occasionally used to extend the SM, e.g., $D_{4}, D_{5}, D_{7}, D_{14}, \Delta(54), \Delta(96)$, and $\Sigma(81)$ have all appeared in the 
literature [56-58]. For a discussion of breaking $\mathrm{SO}(3)$ to dihedral groups see [54]. Further information about the classification of the discrete subgroups of $\mathrm{SU}(3)$ can be found in $[20,59-61]$. In addition products of discrete groups are often employed, where the products often contain $Z_{n}$ factors. For example if the fundamental charge of a U(1) gauge group is $q$, then by breaking a $\mathrm{U}(1)$ with scalar particle of charge $n q$ one arrives at $Z_{n}$. To gauge these cases we can start with a product gauge group and break to the desired discrete group, $G_{1} \times G_{2} \times \ldots \rightarrow \Gamma_{1} \times \Gamma_{2} \times \ldots$. As long as there are no cross terms in the scalar potential, then we can proceed as above. In some cases the cross terms can destabilize the minima, so they must either be eliminated, or dealt with by other means. Results given here could be applied to extend recent work on gauging two Higgs doublet models [62]. Using our results to extend models currently in the literature can solve some existing problems, and the inclusion of new scalars in the spectrum may be of interest, since some may be detectable either directly or indirectly depending on the details of the model. Such phenomenological investigations need to proceed on a model by model basis, and we plan to look at some specific examples in future work.

\section{Acknowledgments}

We have benefited greatly from discussions and correspondences with Christoph Luhn, Alex Merle and Pierre Ramond.

\section{A Branching rules}

In this appendix we present the branching rules for the embeddings of discrete groups into Lie groups used in the paper. The vertical axes label the dimensions of the Lie Group representations, and the horizontal the dimensions of the discrete group representations. We begin with a list of tables.

- Table 1: Scalar mass eigenstates for the SSB pattern $\mathrm{SO}(3) \rightarrow A_{4}$ using a real 7 of $\mathrm{SO}(3)$;

- Table 2: Scalar mass eigenstates for the SSB pattern $\mathrm{SO}(3) \rightarrow S_{4}$ using a real 9 of $\mathrm{SO}(3)$;

- Table 3: Scalar mass eigenstates for the SSB pattern $\mathrm{SO}(3) \rightarrow A_{5}$ using a real $\mathbf{1 3}$ of $\mathrm{SO}(3)$;

- Table 4: Scalar mass eigenstates for the SSB pattern $\mathrm{SU}(2) \rightarrow Q_{6}$ using a complex 7 of $\mathrm{SU}(2)$;

- Table 5: Scalar mass eigenstates for the SSB pattern $\mathrm{SU}(2) \rightarrow T^{\prime}$ using a complex 7 of $\mathrm{SU}(2)$;

- Table 6: Scalar mass eigenstates for the SSB pattern $\mathrm{SU}(2) \rightarrow O^{\prime}$ using a complex 9 of $\mathrm{SU}(2)$; 
- Table 7: Scalar mass eigenstates for the SSB pattern $\mathrm{SU}(2) \rightarrow I^{\prime}$ using a complex 13 of $\mathrm{SU}(2)$;

- Table 8: Scalar mass eigenstates for the SSB pattern $\mathrm{SU}(3) \rightarrow A_{4}$ using a $\mathbf{1 5}$ of $\mathrm{SU}(3)$;

- Table 9: Scalar mass eigenstates for the SSB pattern $\mathrm{SU}(3) \rightarrow T_{7}$ using a $\mathbf{1 5}$ of $\mathrm{SU}(3)$;

- Table 10: Scalar mass eigenstates for the SSB pattern SU(3) $\rightarrow \Delta(27)$ using a 10 of $\mathrm{SU}(3)$;

- Table 11: Scalar mass eigenstates for the SSB pattern $\operatorname{SU}(3) \rightarrow \operatorname{PSL}(2,7)$ using a $\mathbf{1 5}^{\prime}$ of $\mathrm{SU}(3)$;

- Table 12: Numerical results where cubic terms are included for the scalar mass eigenstates of the SSB pattern $\mathrm{SU}(3) \rightarrow \operatorname{PSL}(2,7)$ using a $\mathbf{1 5}^{\prime}$ of $\mathrm{SU}(3)$;

- Table 13: Scalar mass eigenstates for the SSB pattern SU(3) $\rightarrow$ PSL $(2,7)$ using a 28 of SU(3);

- Table 14: Scalar mass eigenstates for the SSB patterns $\mathrm{SO}(3) \rightarrow A_{4}, S_{4}$ and $A_{5}$ using real symmetric tensor irreps of $\mathrm{SO}(3)$;

- Table 15: Scalar mass eigenstates for the SSB patterns $\mathrm{SU}(2) \rightarrow Q_{6}, T^{\prime}, O^{\prime}$ and $I^{\prime}$ using symmetric tensor irreps of $\mathrm{SU}(2)$, i.e., complexified symmetric tensor irreps of $\mathrm{SO}(3)$;

- Table 16: Scalar mass eigenstates for the SSB patterns $\mathrm{SU}(3) \rightarrow A_{4}, T_{7}, \Delta(27)$ and $\operatorname{PSL}(2,7)$ using symmetric tensor irreps of $\mathrm{SU}(3)$, except for the $\mathbf{1 5}$ which is of mixed symmetry;

- Table 17: $\mathrm{SO}(3) \rightarrow A_{4}$;

- Table 18: $\mathrm{SO}(3) \rightarrow S_{4}$;

- Table 19: $\mathrm{SO}(3) \rightarrow A_{5}$;

- Table 20: $\mathrm{SU}(2) \rightarrow Q_{6}$;

- Table 21: $\mathrm{SU}(2) \rightarrow T^{\prime}$;

- Table 22: $\mathrm{SU}(2) \rightarrow O^{\prime}$;

- Table 23: $\mathrm{SU}(2) \rightarrow I^{\prime}$;

- Table 24: $\mathrm{SU}(3) \rightarrow A_{4}$;

- Table 25: $\mathrm{SU}(3) \rightarrow T_{7}$;

- Table 26: $\mathrm{SU}(3) \rightarrow \Delta(27)$.

- Table 27: $\mathrm{SU}(3) \rightarrow \operatorname{PSL}(2,7)$ 


\begin{tabular}{|c|cccc|}
\hline Dimension & $\mathbf{1}_{\mathbf{1}}$ & $\mathbf{1}_{\mathbf{2}}$ & $\mathbf{1}_{\mathbf{3}}$ & $\mathbf{3}$ \\
\hline $\mathbf{3}$ & 0 & 0 & 0 & 1 \\
$\mathbf{5}$ & 0 & 1 & 1 & 1 \\
$\mathbf{7}$ & 1 & 0 & 0 & 2 \\
$\mathbf{9}$ & 1 & 1 & 1 & 2 \\
$\mathbf{1 1}$ & 0 & 1 & 1 & 3 \\
\hline
\end{tabular}

Table 17. $\mathrm{SO}(3) \rightarrow A_{4}$.

\begin{tabular}{|c|ccccc|}
\hline Dimension & $\mathbf{1}$ & $\mathbf{3}$ & $\mathbf{3}$ & $\mathbf{4}$ & $\mathbf{5}$ \\
\hline $\mathbf{3}$ & 0 & 0 & 1 & 0 & 0 \\
$\mathbf{5}$ & 0 & 0 & 0 & 0 & 1 \\
$\mathbf{7}$ & 0 & 1 & 0 & 1 & 0 \\
$\mathbf{9}$ & 0 & 0 & 0 & 1 & 1 \\
$\mathbf{1 1}$ & 0 & 1 & 1 & 0 & 1 \\
$\mathbf{1 3}$ & 1 & 0 & 1 & 1 & 1 \\
\hline
\end{tabular}

Table 19. $\mathrm{SO}(3) \rightarrow A_{5}$.

\begin{tabular}{|c|ccccccc|}
\hline Dimension & $\mathbf{1}_{\mathbf{1}}$ & $\mathbf{1}_{\mathbf{2}}$ & $\mathbf{1}_{\mathbf{3}}$ & $\mathbf{2}_{\mathbf{1}}$ & $\mathbf{2}_{\mathbf{2}}$ & $\mathbf{2}_{\mathbf{3}}$ & $\mathbf{3}$ \\
\hline $\mathbf{2}$ & 0 & 0 & 0 & 1 & 0 & 0 & 0 \\
$\mathbf{3}$ & 0 & 0 & 0 & 0 & 0 & 0 & 1 \\
$\mathbf{4}$ & 0 & 0 & 0 & 0 & 1 & 1 & 0 \\
$\mathbf{5}$ & 0 & 1 & 1 & 0 & 0 & 0 & 1 \\
$\mathbf{6}$ & 0 & 0 & 0 & 1 & 1 & 1 & 0 \\
$\mathbf{7}$ & 1 & 0 & 0 & 0 & 0 & 0 & 2 \\
$\mathbf{8}$ & 0 & 0 & 0 & 2 & 1 & 1 & 0 \\
$\mathbf{9}$ & 1 & 1 & 1 & 0 & 0 & 0 & 2 \\
$\mathbf{1 0}$ & 0 & 0 & 0 & 1 & 2 & 2 & 0 \\
$\mathbf{1 1}$ & 0 & 1 & 1 & 0 & 0 & 0 & 3 \\
\hline
\end{tabular}

Table 21. $\mathrm{SU}(2) \rightarrow T^{\prime}$.

\begin{tabular}{|c|ccccccccc|}
\hline Dimension & $\mathbf{1}_{\mathbf{1}}$ & $\mathbf{2}_{\mathbf{2}}$ & $\mathbf{2}_{\mathbf{3}}$ & $\mathbf{3}_{\mathbf{1}}$ & $\mathbf{3}_{\mathbf{2}}$ & $\mathbf{4}_{\mathbf{1}}$ & $\mathbf{4}_{\mathbf{2}}$ & $\mathbf{5}$ & $\mathbf{6}$ \\
\hline $\mathbf{2}$ & 0 & 1 & 0 & 0 & 0 & 0 & 0 & 0 & 0 \\
$\mathbf{3}$ & 0 & 0 & 0 & 0 & 1 & 0 & 0 & 0 & 0 \\
$\mathbf{4}$ & 0 & 0 & 0 & 0 & 0 & 0 & 1 & 0 & 0 \\
$\mathbf{5}$ & 0 & 0 & 0 & 0 & 0 & 0 & 0 & 1 & 0 \\
$\mathbf{6}$ & 0 & 0 & 0 & 0 & 0 & 0 & 0 & 0 & 1 \\
$\mathbf{7}$ & 0 & 0 & 0 & 1 & 0 & 1 & 0 & 0 & 0 \\
$\mathbf{8}$ & 0 & 0 & 1 & 0 & 0 & 0 & 0 & 0 & 1 \\
$\mathbf{9}$ & 0 & 0 & 0 & 0 & 0 & 1 & 0 & 1 & 0 \\
$\mathbf{1 0}$ & 0 & 1 & 0 & 0 & 0 & 0 & 1 & 0 & 1 \\
$\mathbf{1 1}$ & 0 & 0 & 0 & 1 & 1 & 0 & 0 & 1 & 0 \\
$\mathbf{1 2}$ & 0 & 1 & 0 & 0 & 0 & 0 & 1 & 0 & 1 \\
$\mathbf{1 3}$ & 1 & 0 & 0 & 0 & 1 & 1 & 0 & 1 & 0 \\
\hline
\end{tabular}

Table 23. $\mathrm{SU}(2) \rightarrow I^{\prime}$.

\begin{tabular}{|c|ccccc|}
\hline Dimension & $\mathbf{1}_{\mathbf{1}}$ & $\mathbf{1}_{\mathbf{2}}$ & $\mathbf{2}$ & $\mathbf{3}_{\mathbf{1}}$ & $\mathbf{3}_{\mathbf{2}}$ \\
\hline $\mathbf{3}$ & 0 & 0 & 0 & 1 & 0 \\
$\mathbf{5}$ & 0 & 0 & 1 & 0 & 1 \\
$\mathbf{7}$ & 0 & 1 & 0 & 1 & 1 \\
$\mathbf{9}$ & 1 & 0 & 1 & 1 & 1 \\
$\mathbf{1 1}$ & 0 & 0 & 1 & 2 & 1 \\
\hline
\end{tabular}

Table 18. $\mathrm{SO}(3) \rightarrow S_{4}$.

\begin{tabular}{|c|cccccc|}
\hline Dimension & $\mathbf{1}_{\mathbf{1}}$ & $\mathbf{1}_{\mathbf{2}}$ & $\mathbf{1}_{\mathbf{3}}$ & $\mathbf{1}_{\mathbf{4}}$ & $\mathbf{2}_{\mathbf{1}}$ & $\mathbf{2}_{\mathbf{2}}$ \\
\hline $\mathbf{2}$ & 0 & 0 & 0 & 0 & 1 & 0 \\
$\mathbf{3}$ & 0 & 1 & 0 & 0 & 0 & 1 \\
$\mathbf{4}$ & 0 & 0 & 1 & 1 & 1 & 0 \\
$\mathbf{5}$ & 1 & 0 & 0 & 0 & 0 & 2 \\
$\mathbf{6}$ & 0 & 0 & 1 & 1 & 2 & 0 \\
$\mathbf{7}$ & 1 & 2 & 0 & 0 & 0 & 2 \\
$\mathbf{8}$ & 0 & 0 & 1 & 1 & 3 & 0 \\
$\mathbf{9}$ & 2 & 1 & 0 & 0 & 0 & 3 \\
$\mathbf{1 0}$ & 0 & 0 & 2 & 2 & 3 & 0 \\
$\mathbf{1 1}$ & 1 & 2 & 0 & 0 & 0 & 4 \\
\hline
\end{tabular}

Table 20. $\mathrm{SU}(2) \rightarrow Q_{6}$.

\begin{tabular}{|c|cccccccc|}
\hline Dimension & $\mathbf{1}_{\mathbf{1}}$ & $\mathbf{1}_{\mathbf{2}}$ & $\mathbf{2}_{\mathbf{1}}$ & $\mathbf{2}_{\mathbf{2}}$ & $\mathbf{2}_{\mathbf{3}}$ & $\mathbf{3}_{\mathbf{1}}$ & $\mathbf{3}_{\mathbf{2}}$ & $\mathbf{4}$ \\
\hline $\mathbf{2}$ & 0 & 0 & 0 & 1 & 0 & 0 & 0 & 0 \\
$\mathbf{3}$ & 0 & 0 & 0 & 0 & 0 & 0 & 1 & 0 \\
$\mathbf{4}$ & 0 & 0 & 0 & 0 & 0 & 0 & 0 & 1 \\
$\mathbf{5}$ & 0 & 0 & 1 & 0 & 0 & 1 & 0 & 0 \\
$\mathbf{6}$ & 0 & 0 & 0 & 0 & 1 & 0 & 0 & 1 \\
$\mathbf{7}$ & 0 & 1 & 0 & 0 & 0 & 1 & 1 & 0 \\
$\mathbf{8}$ & 0 & 0 & 0 & 1 & 1 & 0 & 0 & 1 \\
$\mathbf{9}$ & 1 & 0 & 1 & 0 & 0 & 1 & 1 & 0 \\
$\mathbf{1 0}$ & 0 & 0 & 0 & 1 & 0 & 0 & 0 & 2 \\
$\mathbf{1 1}$ & 0 & 0 & 1 & 0 & 0 & 1 & 2 & 0 \\
\hline
\end{tabular}

Table 22. $\mathrm{SU}(2) \rightarrow O^{\prime}$.

\begin{tabular}{|c|cccc|}
\hline Dimension & $\mathbf{1}_{\mathbf{1}}$ & $\mathbf{1}_{\mathbf{2}}$ & $\mathbf{1}_{\mathbf{3}}$ & $\mathbf{3}$ \\
\hline $\mathbf{3}$ & 0 & 0 & 0 & 1 \\
$\mathbf{6}$ & 1 & 1 & 1 & 1 \\
$\mathbf{8}$ & 0 & 1 & 1 & 2 \\
$\mathbf{1 0}$ & 1 & 0 & 0 & 3 \\
$\mathbf{1 5}$ & 1 & 1 & 1 & 4 \\
$\mathbf{1 5}$ & 2 & 2 & 2 & 3 \\
$\mathbf{2 1}$ & 1 & 1 & 1 & 6 \\
$\mathbf{2 4}$ & 2 & 2 & 2 & 6 \\
$\mathbf{2 7}$ & 3 & 3 & 3 & 6 \\
\hline
\end{tabular}

Table 24. SU(3) $\rightarrow A_{4}$. 


\begin{tabular}{|c|ccccc|}
\hline Dimension & $\mathbf{1}_{\mathbf{1}}$ & $\mathbf{1}_{\mathbf{2}}$ & $\mathbf{1}_{\mathbf{3}}$ & $\mathbf{3}_{\mathbf{1}}$ & $\mathbf{3}_{\mathbf{2}}$ \\
\hline $\mathbf{3}$ & 0 & 0 & 0 & 1 & 0 \\
$\mathbf{6}$ & 0 & 0 & 0 & 1 & 1 \\
$\mathbf{8}$ & 0 & 1 & 1 & 1 & 1 \\
$\mathbf{1 0}$ & 1 & 0 & 0 & 1 & 2 \\
$\mathbf{1 5}$ & 1 & 1 & 1 & 2 & 2 \\
$\mathbf{1 5}^{\prime}$ & 1 & 1 & 1 & 2 & 2 \\
$\mathbf{2 1}$ & 1 & 1 & 1 & 3 & 3 \\
$\mathbf{2 4}$ & 1 & 1 & 1 & 4 & 3 \\
$\mathbf{2 7}$ & 1 & 1 & 1 & 4 & 4 \\
\hline
\end{tabular}

Table 25. $\mathrm{SU}(3) \rightarrow T_{7}$.

\begin{tabular}{|c|ccccccccccc|}
\hline Dimension & $\mathbf{1}_{\mathbf{1}}$ & $\mathbf{1}_{\mathbf{2}}$ & $\mathbf{1}_{\mathbf{3}}$ & $\mathbf{1}_{\mathbf{4}}$ & $\mathbf{1}_{\mathbf{5}}$ & $\mathbf{1}_{\mathbf{6}}$ & $\mathbf{1}_{\mathbf{7}}$ & $\mathbf{1}_{\mathbf{8}}$ & $\mathbf{1}_{\mathbf{9}}$ & $\mathbf{3}_{\mathbf{1}}$ & $\mathbf{3}_{\mathbf{2}}$ \\
\hline $\mathbf{3}$ & 0 & 0 & 0 & 0 & 0 & 0 & 0 & 0 & 0 & 1 & 0 \\
$\mathbf{6}$ & 0 & 0 & 0 & 0 & 0 & 0 & 0 & 0 & 0 & 0 & 2 \\
$\mathbf{8}$ & 0 & 1 & 1 & 1 & 1 & 1 & 1 & 1 & 1 & 0 & 0 \\
$\mathbf{1 0}$ & 2 & 1 & 1 & 1 & 1 & 1 & 1 & 1 & 1 & 0 & 0 \\
$\mathbf{1 5}$ & 0 & 0 & 0 & 0 & 0 & 0 & 0 & 0 & 0 & 5 & 0 \\
$\mathbf{1 5}$ & 0 & 0 & 0 & 0 & 0 & 0 & 0 & 0 & 0 & 5 & 0 \\
$\mathbf{2 1}$ & 0 & 0 & 0 & 0 & 0 & 0 & 0 & 0 & 0 & 0 & 7 \\
$\mathbf{2 4}$ & 0 & 0 & 0 & 0 & 0 & 0 & 0 & 0 & 0 & 0 & 8 \\
$\mathbf{2 7}$ & 3 & 3 & 3 & 3 & 3 & 3 & 3 & 3 & 3 & 0 & 0 \\
\hline
\end{tabular}

Table 26. $\mathrm{SU}(3) \rightarrow \Delta(27)$.

\begin{tabular}{|c|cccccc|}
\hline Dimension & $\mathbf{1}$ & $\mathbf{3}_{\mathbf{2}}$ & $\mathbf{3}_{\mathbf{2}}$ & $\mathbf{6}$ & $\mathbf{7}$ & $\mathbf{8}$ \\
\hline $\mathbf{3}$ & 0 & 1 & 0 & 0 & 0 & 0 \\
$\mathbf{6}$ & 0 & 0 & 0 & 1 & 0 & 0 \\
$\mathbf{8}$ & 0 & 0 & 0 & 0 & 0 & 1 \\
$\mathbf{1 0}$ & 0 & 0 & 1 & 0 & 1 & 0 \\
$\mathbf{1 5}$ & 0 & 0 & 0 & 0 & 1 & 1 \\
$\mathbf{1 5}^{\prime}$ & 1 & 0 & 0 & 1 & 0 & 1 \\
$\mathbf{2 1}$ & 0 & 1 & 1 & 0 & 1 & 1 \\
$\mathbf{2 4}$ & 0 & 1 & 0 & 1 & 1 & 1 \\
$\mathbf{2 7}$ & 0 & 0 & 0 & 2 & 1 & 1 \\
$\mathbf{2 8}$ & 1 & 0 & 0 & 2 & 1 & 1 \\
\hline
\end{tabular}

Table 27. $\mathrm{SU}(3) \rightarrow \operatorname{PSL}(2,7)$.

Open Access. This article is distributed under the terms of the Creative Commons Attribution License (CC-BY 4.0), which permits any use, distribution and reproduction in any medium, provided the original author(s) and source are credited.

\section{References}

[1] S. Pakvasa and H. Sugawara, Discrete symmetry and Cabibbo angle, Phys. Lett. B 73 (1978) 61.

[2] D. Wyler, Discrete symmetries in the six quark $\mathrm{SU}(2) \times \mathrm{U}(1)$ model, Phys. Rev. D 19 (1979) 3369 [INSPIRE].

[3] E. Ma and G. Rajasekaran, Softly broken $A_{4}$ symmetry for nearly degenerate neutrino masses, Phys. Rev. D 64 (2001) 113012 [hep-ph/0106291] [INSPIRE].

[4] K.S. Babu, E. Ma and J.W.F. Valle, Underlying $A_{4}$ symmetry for the neutrino mass matrix and the quark mixing matrix, Phys. Lett. B 552 (2003) 207 [hep-ph/0206292] [INSPIRE].

[5] P.H. Frampton and T.W. Kephart, Simple nonAbelian finite flavor groups and fermion masses, Int. J. Mod. Phys. A 10 (1995) 4689 [hep-ph/9409330] [INSPIRE].

[6] H. Ishimori, T. Kobayashi, H. Ohki, Y. Shimizu, H. Okada and M. Tanimoto, Non-abelian discrete symmetries in particle physics, Prog. Theor. Phys. Suppl. 183 (2010) 1 [arXiv: 1003.3552] [INSPIRE].

[7] G. Altarelli and F. Feruglio, Discrete flavor symmetries and models of neutrino mixing, Rev. Mod. Phys. 82 (2010) 2701 [arXiv: 1002.0211] [INSPIRE]. 
[8] S.F. King and C. Luhn, Neutrino mass and mixing with discrete symmetry, Rept. Prog. Phys. 76 (2013) 056201 [arXiv: 1301.1340] [INSPIRE].

[9] S.F. King, A. Merle, S. Morisi, Y. Shimizu and M. Tanimoto, Neutrino mass and mixing: from theory to experiment, New J. Phys. 16 (2014) 045018 [arXiv:1402.4271] [INSPIRE].

[10] L.M. Krauss and F. Wilczek, Discrete gauge symmetry in continuum theories, Phys. Rev. Lett. 62 (1989) 1221 [INSPIRE].

[11] R. Holman et al., Solutions to the strong CP problem in a world with gravity, Phys. Lett. $\mathbf{B}$ 282 (1992) 132 [hep-ph/9203206] [INSPIRE];

[12] M. Kamionkowski and J. March-Russell, Planck scale physics and the Peccei-Quinn mechanism, Phys. Lett. B 282 (1992) 137 [hep-th/9202003] [INSPIRE].

[13] S.M. Barr and D. Seckel, Planck scale corrections to axion models, Phys. Rev. D 46 (1992) 539 [INSPIRE].

[14] C. Luhn and P. Ramond, Anomaly conditions for non-abelian finite family symmetries, JHEP 07 (2008) 085 [arXiv: 0805.1736] [INSPIRE].

[15] M.B. Hindmarsh and T.W.B. Kibble, Cosmic strings, Rept. Prog. Phys. 58 (1995) 477 [hep-ph/9411342] [INSPIRE].

[16] O. Reynolds, On the dynamical theory of incompressible viscous fluids and the determination of the criterion, Phil. Trans. Roy. Soc. A 186 (1895) 123.

[17] B. Sturmfels, Algorithms in invariant theory, $2^{\text {nd }}$ ed., Springer, Germmany (2008).

[18] T. Molien, Uber die Invarianten der linearen Substitutionsgruppen, Sitz. König Preuss. Akad. Wiss. 52 (1897) 1152.

[19] C. Luhn, Spontaneous breaking of SU(3) to finite family symmetries: a pedestrian's approach, JHEP 03 (2011) 108 [arXiv:1101.2417] [INSPIRE].

[20] A. Merle and R. Zwicky, Explicit and spontaneous breaking of SU(3) into its finite subgroups, JHEP 02 (2012) 128 [arXiv:1110.4891] [INSPIRE].

[21] M. Fallbacher, Breaking classical Lie groups to finite subgroups - An automated approach, Nucl. Phys. B 898 (2015) 229 [arXiv:1506.03677] [INSPIRE].

[22] GAP - Groups, Algorithms, Programming - A system for computational discrete algebra, version 4.8.5 (2016).

[23] P.H. Frampton, T.W. Kephart and R.M. Rohm, A note on embedding nonabelian finite flavor groups in continuous groups, Phys. Lett. B 679 (2009) 478 [arXiv:0904.0420] [INSPIRE].

[24] J. Berger and Y. Grossman, Model of leptons from $\mathrm{SO}(3) \rightarrow A_{4}$, JHEP 02 (2010) 071 [arXiv: 0910.4392] [INSPIRE].

[25] E. Ma, Neutrino mixing: $A_{4}$ variations, Phys. Lett. B 752 (2016) 198 [arXiv:1510.02501] [INSPIRE].

[26] G. Altarelli, F. Feruglio and C. Hagedorn, A SUSY SU(5) grand unified model of tri-bimaximal mixing from $A_{4}$, JHEP 03 (2008) 052 [arXiv: 0802.0090] [INSPIRE].

[27] S.F. King and C. Luhn, $A_{4}$ models of tri-bimaximal-reactor mixing, JHEP 03 (2012) 036 [arXiv:1112.1959] [INSPIRE].

[28] P.M. Ferreira, L. Lavoura and P.O. Ludl, A new $A_{4}$ model for lepton mixing, Phys. Lett. B 726 (2013) 767 [arXiv:1306.1500] [INSPIRE]. 
[29] P. Cvitanovic, Group theory for Feynman diagrams in non-Abelian gauge theories, Phys. Rev. D 14 (1976) 1536 [INSPIRE].

[30] C. Hagedorn, M. Lindner and R.N. Mohapatra, $S_{4}$ flavor symmetry and fermion masses: towards a grand unified theory of flavor, JHEP 06 (2006) 042 [hep-ph/0602244] [INSPIRE].

[31] C. Hagedorn, S.F. King and C. Luhn, A SUSY GUT of Flavour with $S^{4} \times \mathrm{SU}(5)$ to NLO, JHEP 06 (2010) 048 [arXiv: 1003.4249] [INSPIRE].

[32] L.L. Everett and A.J. Stuart, Icosahedral $\left(A_{5}\right)$ family symmetry and the golden ratio prediction for solar neutrino mixing, Phys. Rev. D 79 (2009) 085005 [arXiv:0812.1057] [INSPIRE].

[33] F. Feruglio and A. Paris, The golden ratio prediction for the solar angle from a natural model with $A_{5}$ flavour symmetry, JHEP 03 (2011) 101 [arXiv:1101.0393] [INSPIRE].

[34] G.-J. Ding, L.L. Everett and A.J. Stuart, Golden ratio neutrino mixing and $A_{5}$ flavor symmetry, Nucl. Phys. B 857 (2012) 219 [arXiv:1110.1688] [INSPIRE].

[35] C.-S. Chen, T.W. Kephart and T.-C. Yuan, An $A_{5}$ model of four lepton generations, JHEP 04 (2011) 015 [arXiv: 1011.3199] [INSPIRE].

[36] P.H. Frampton and T.W. Kephart, Minimal family unification, Phys. Rev. D 51 (1995) R1 [hep-ph/9409324] [INSPIRE].

[37] P.H. Frampton and A. Rasin, NonAbelian discrete symmetries, fermion mass textures and large neutrino mixing, Phys. Lett. B 478 (2000) 424 [hep-ph/9910522] [INSPIRE].

[38] A. Aranda, C.D. Carone and R.F. Lebed, U(2) flavor physics without U(2) symmetry, Phys. Lett. B 474 (2000) 170 [hep-ph/9910392] [INSPIRE].

[39] M.-C. Chen and K.T. Mahanthappa, CKM and tri-bimaximal MNS matrices in a $\mathrm{SU}(5) \times{ }^{(d)} T$ model, Phys. Lett. B 652 (2007) 34 [arXiv:0705.0714] [INSPIRE].

[40] P.H. Frampton and T.W. Kephart, Flavor symmetry for quarks and leptons, JHEP 09 (2007) 110 [arXiv:0706.1186] [INSPIRE].

[41] P.H. Frampton, T.W. Kephart and S. Matsuzaki, Simplified renormalizable T-prime model for tribimaximal mixing and Cabibbo angle, Phys. Rev. D 78 (2008) 073004 [arXiv:0807.4713] [INSPIRE].

[42] P.H. Frampton, C.M. Ho, T.W. Kephart and S. Matsuzaki, LHC Higgs production and decay in the T' model, Phys. Rev. D 82 (2010) 113007 [arXiv: 1009.0307] [InSPIRE].

[43] A. Natale, A radiative model of quark masses with binary tetrahedral symmetry, Nucl. Phys. B 914 (2017) 201 [arXiv: 1608.06999] [INSPIRE].

[44] C.D. Carone, S. Chaurasia and S. Vasquez, Flavor from the double tetrahedral group without supersymmetry, Phys. Rev. D 95 (2017) 015025 [arXiv:1611.00784] [INSPIRE].

[45] L.L. Everett and A.J. Stuart, The double cover of the icosahedral symmetry group and quark mass textures, Phys. Lett. B 698 (2011) 131 [arXiv:1011.4928] [INSPIRE].

[46] C.-S. Chen, T.W. Kephart and T.-C. Yuan, Binary icosahedral flavor symmetry for four generations of quarks and leptons, PTEP 2013 (2013) 103B01 [arXiv:1110.6233] [INSPIRE].

[47] C. Luhn, S. Nasri and P. Ramond, Tri-bimaximal neutrino mixing and the family symmetry semidirect product of $Z_{7}$ and $Z_{3}$, Phys. Lett. B 652 (2007) 27 [arXiv:0706.2341] [INSPIRE]. 
[48] J. Kile, M.J. Pérez, P. Ramond and J. Zhang, $\theta_{13}$ and the flavor ring, Phys. Rev. D 90 (2014) 013004 [arXiv: 1403.6136] [INSPIRE].

[49] V.V. Vien, $T_{7}$ flavor symmetry scheme for understanding neutrino mass and mixing in 3-3-1 model with neutral leptons, Mod. Phys. Lett. A 29 (2014) 28 [arXiv:1508. 02585] [InSPIRE].

[50] V.V. Vien and H.N. Long, Lepton mass and mixing in a simple extension of the Standard Model based on $T^{7}$ flavor symmetry, arXiv:1609.03895 [INSPIRE].

[51] V.V. Vien, A.E. Cárcamo Hernández and H.N. Long, The $\Delta(27)$ flavor 3-3-1 model with neutral leptons, Nucl. Phys. B 913 (2016) 792 [arXiv:1601.03300] [InSPIRE].

[52] P.M. Ferreira, W. Grimus, L. Lavoura and P.O. Ludl, Maximal CP-violation in lepton mixing from a model with $\Delta(27)$ flavour Symmetry, JHEP 09 (2012) 128 [arXiv:1206.7072] [INSPIRE].

[53] G. Chen, M.J. Pérez and P. Ramond, Neutrino masses, the $\mu$-term and $\mathrm{PSL}_{2}(7)$, Phys. Rev. D 92 (2015) 076006 [arXiv: 1412.6107] [InSPIRE].

[54] M. Koca, R. Koc and H. Tutunculer, Explicit breaking of $\mathrm{SO}(3)$ with Higgs fields in the representations $l=2$ and $l=3$, Int. J. Mod. Phys. A 18 (2003) 4817 [hep-ph/0410270] [INSPIRE].

[55] C. Luhn, private communication.

[56] C. Hagedorn, M.A. Schmidt and A. Yu. Smirnov, Lepton mixing and cancellation of the Dirac mass hierarchy in $\mathrm{SO}(10)$ GUTs with flavor symmetries $T^{7}$ and $\Sigma(81)$, Phys. Rev. D 79 (2009) 036002 [arXiv:0811.2955] [INSPIRE].

[57] S.F. King, C. Luhn and A.J. Stuart, A grand $\Delta(96) \times \mathrm{SU}(5)$ flavour model, Nucl. Phys. B 867 (2013) 203 [arXiv: 1207.5741] [INSPIRE].

[58] V.V. Vien and H.N. Long, Quark masses and mixings in the 3-3-1 model with neutral leptons based on $D_{4}$ flavor symmetry, J. Korean Phys. Soc. 66 (2015) 1809 [arXiv:1408.4333] [INSPIRE].

[59] P.O. Ludl, On the finite subgroups of U(3) of order smaller than 512, J. Phys. A 43 (2010) 395204 [Erratum ibid. A 44 (2011) 139501] [arXiv:1006.1479] [INSPIRE].

[60] W. Grimus and P.O. Ludl, Finite flavour groups of fermions, J. Phys. A 45 (2012) 233001 [arXiv: 1110.6376] [INSPIRE].

[61] P.O. Ludl, Comments on the classification of the finite subgroups of SU(3), J. Phys. A 44 (2011) 255204 [Erratum ibid. A 45 (2012) 069502] [arXiv:1101.2308] [INSPIRE].

[62] W.-C. Huang, Y.-L.S. Tsai and T.-C. Yuan, G2HDM: gauged two Higgs doublet model, JHEP 04 (2016) 019 [arXiv: 1512.00229] [INSPIRE]. 